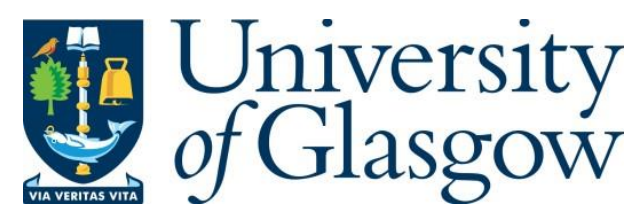

Zhang, H., Tan, S. Y. and Seow, C. K. (2019) TOA-based indoor localization and tracking with inaccurate floor plan map via MRMSC-PHD filter. IEEE Sensors Journal, 19(21), pp. 9869-9882.

There may be differences between this version and the published version. You are advised to consult the publisher's version if you wish to cite from it.

http://eprints.gla.ac.uk/200579/

Deposited on: 18 October 2019

Enlighten - Research publications by members of the University of Glasgow http://eprints.gla.ac.uk 


\title{
TOA based Indoor Localization and Tracking with Inaccurate Floor Plan Map via MRMSC-PHD Filter
}

\author{
Heng Zhang, Soon Yim Tan, Member, IEEE and Chee Kiat Seow, Senior Member, IEEE
}

\begin{abstract}
This paper proposes a novel indoor localization scheme to jointly track a mobile device (MD) and update an inaccurate floor plan map using the time-of-arrival measured at multiple reference devices (RDs). By modeling the floor plan map as a collection of map features, the map and MD position can be jointly estimated via a multi-RD single-cluster probability hypothesis density (MSC-PHD) filter. Conventional MSC-PHD filters assume that each map feature generates at most one measurement for each RD. If single reflections of the detected signal are considered as measurements generated by map features, then higher-order reflections, which also carry information on the MD and map features, must be treated as clutter. The proposed scheme incorporates multiple reflections by treating them as virtual single reflections reflected from inaccurate map features and traces them to the corresponding virtual RDs (VRDs), referred to as a multi-reflection-incorporating MSCPHD (MRMSC-PHD) filter. The complexity of using multiple reflection paths arises from the inaccuracy of the VRD location due to inaccuracy in the map features. Numerical results show that these multiple reflection paths can be modeled statistically as a Gaussian distribution. A computationally tractable implementation combining a new greedy partitioning scheme and a particle-Gaussian mixture filter is presented. A novel mapping error metric is then proposed to evaluate the estimated map?s accuracy for plane surfaces. Simulation and experimental results show that our proposed MRMSC-PHD filter outperforms existing MSC-PHD filters by up to $95 \%$ in terms of average localization and by up to $90 \%$ in terms of mapping accuracy.
\end{abstract}

Index Terms-Localization, tracking, time-of-arrival, multireflections, multi-RD single-cluster probability hypothesis density filter, mapping error.

\section{INTRODUCTION}

$\mathbf{I}$ NDOOR localization and tracking algorithms for wireless devices have been widely studied. The frameworks of these algorithms are based on measurements such as received signal strength (RSS) and time-of-arrival (TOA) [1]-[3]. RSS is easy to measure using wireless devices, but struggles to achieve high-ranging accuracy due to severe multipath overlapping in indoor environments [1]. Fingerprinting, which utilizes position-dependent fingerprints to localize and track a mobile device (MD), requires an offline fingerprint collection step to build a database [4]. TOA can provide highly accurate path length information, but requires time synchronization between the reference device (RD) and the MD. To resolve

Manuscript received 20-Sep-2019

H. Zhang and S. Y. Tan are with the School of Electrical and Electronic Engineering, Nanyang Technological University, Singapore, 639798 (e-mail: hzhang019@e.ntu.edu.sg; esytan@ntu.edu.sg).

C. K. Seow is with the School of Computing Science, University of Glasgow, Sir Alwyn Williams Building, Glasgow G12 8RZ, Scotland, United Kingdom. (e-mail: CheeKiat.Seow@glasgow.ac.uk) the synchronization issue, a two-way ranging method can be used, as shown in [5].

To overcome multipath fading, conventional algorithms try to identify and either mitigate or discard multipath components [6], [7]. Recently, algorithms have been developed to utilize multipath components instead of mitigating or discarding them to assist in localizing and tracking MDs, such as mapaided localization algorithms. Such methods utilize an accurate floor plan map to treat multipaths as virtual line-of-sight (LOS) paths traced to corresponding virtual RDs (VRDs) to localize and track an MD [8]-[12]. However, in most indoor cases, an accurate floor plan is unavailable, which necessitates joint estimation of the MD and the map. If the map is modeled as a collection of map features, such as lines, corners, and points, it can be jointly estimated with the MD location. In indoor environments, diffractions from point scatterers and corners are generally weaker than multiple reflections from planar reflectors such as walls, floors, and ceilings [13]. Therefore, this paper treats planar reflectors as the only type of map feature; all of the measurements generated by point scatterers and corners are considered clutter.

The main issue in indoor localization and tracking using multipath components is the alleviation of measurement origin uncertainty, which is an association process that matches measurements with estimated path lengths according to prior information about the MD and map features [14]. Two types of algorithms can be used to address this issue. One type is based on a data association (DA) framework; such algorithms include the joint probabilistic data association filter (JPDAF) [14][17] and the multiple hypothesis tracking filter (MHTF) [18][21]. In [17], a tracking scheme is proposed by modeling both LOS and reflections and using JPDAF to associate measurements with their corresponding path origins. In [20], a tracking and mapping algorithm is proposed that models LOS, reflections, and point scattering paths and uses MHTF to associate measurements with their path origins. The results of these methods are sensitive to DA uncertainty [22]. The other type is based on a random finite set (RFS) Bayesian framework; such algorithms include the Rao-Blackwellized probability hypothesis density (RB-PHD) filter [23], [24] and single cluster PHD (SC-PHD) filter [25]-[28]. These methods involve recursively propagating the PHD of map features (the first-order statistics of the RFS-modeled map features) and full distribution of the MD [29]. Compared with DA-based algorithms, the RFS-based framework has better accuracy and stability because these algorithms incorporate DA uncertainty and an unknown number of map features [23], [30], [31]. In addition, the RB-PHD filter assumes that the prior and poste- 
rior distributions of map features are both Poisson processes, whereas the SC-PHD filter only makes this assumption for the prior distribution [26]. The SC-PHD filter models the MD and map features as a doubly stochastic point process, where the state of the map features is conditional on the state of the MD [26], [28]. The original SC-PHD filter was proposed in [26], where only backscattering paths were considered, and they were modeled as a Poisson point process. In [27], an SC-PHD filter is proposed in which both the LOS path and single reflection paths are modeled as a Poisson point process. In [25], an SC-PHD filter is proposed that models the LOS path as a Bernoulli point process and single reflection paths as a Poisson point process.

To the best of our knowledge, all SC-PHD filters assume that each map feature generates at most one measurement. Thus, if single reflections are considered effective measurements, then higher-order reflections can only be considered clutter. However, higher-order reflections carry information on the MD and map features that could improve the accuracy of localization and mapping. In an indoor multipath environment, double and higher-order reflections are common [32]. If such reflection paths can be modeled in the SC-PHD filter, both mapping and localization accuracy will be improved. Inspired by the multi-detection PHD filter proposed in [33], the multiple reflection issue can be considered a virtual multi-detection problem. However, to incorporate multiple reflections in the SC-PHD filter, it is necessary to decouple the map features involved in multiple reflections. The associations among map features can be decoupled by considering each multiple reflection as a virtual single reflection generated by a single map feature. Note that multi-detection in this paper differs from extended object tracking. In the latter, the extended object is modeled as a collection of point scatterers, and the generated multiple measurements are mainly backscattering and are spatially close to each other [34]. In this paper, the map features are modeled as separate plane reflectors. Multiple measurements per feature indicate multipath propagation involving more than one object. In this case, the multiple measurements are not necessarily spatially close to each other: for example, the single and double reflections from the same reflector may be far apart.

Furthermore, the multi-RD SC-PHD (MSC-PHD) filter is computationally intractable because the number of partitions of the measurements, and the associations of the measurements in each partition, increase combinatorically [33]. In [35], an iterated-corrector approximation method is proposed to address this issue. The iterated-corrector approximation iteratively updates the PHD of the MD and the map features using measurements from a single RD each time. The updated PHD of the MD and map features from the previous RD provides the predicted PHD for the next RD, and is updated again using the measurements from the next RD. However, it has been reported that the estimation results depend on the order in which the RDs are processed [36]. An alternative way to mitigate the computational load is to directly reduce the number of partitions and associations in each partition [33], [34], [37]-[39]. In [37], a distance partition method is proposed for extended target tracking. It groups closed measurements in the same cell and then partitions the cells. In [33], an $l_{\text {max }}$ partition method is proposed for multi-detection tracking. It restricts the number of elements in each measurement subset to be no larger than the number of propagation paths $l$. In [34], a twostep partition algorithm including a distance partition and an $l_{\text {max }}$ partition is proposed. By using a distance partition with a self-defined multi-detection distance, measurements can be initially partitioned. Then, by applying the $l_{\text {max }}$ partition to the partitioned measurement set, the number of resulting partitions can be further reduced. In [38], [39], a greedy partition method is proposed to reduce the number of partitions. It considers only a certain number of partitions with the highest weight at every step and discards the others. However, these methods were proposed for tracking situations in which the MD is the only source of uncertainties. In this paper, the uncertainties of map features are also taken into account.

To the best of our knowledge, no study has provided a definition of the error in estimated map features for non-point scatterers, such as planes. The conventional Euclidean-based metric, which is used to define the error in point scatterers [23], [26], cannot be used to define the error in planes in $\varphi-\rho$ space for two reasons: the units of $\varphi$ and $\rho$ are different, and the Euclidean distance between two planes in $\varphi-\rho$ space is dependent on the origin of the coordinates. And the distance between the estimated and expected VRD is not appropriate to be used to determine the error in the estimated map features, as shown in Appendix B. Therefore, it is important to construct an error metric to resolve these issues.

In short, this paper proposes a novel, first-of-its-kind mapping error metric for planar scatterers to evaluate the accuracy of the estimated map features. In addition, a novel virtual single reflection path model to handle multiple reflections is proposed, and a multi-reflection-incorporating MSC-PHD filter is formulated, which is referred to as an MRMSC-PHD filter. The proposed MRMSC-PHD filter incorporates the LOS path and single, double, triple, and higher-order reflection paths. To reduce the complexity of implementing the MSCPHD filter, a new greedy measurement partition approach is also designed to account for the uncertainties of both the MD and map features.

The remainder of this paper is organized as follows. The multipath propagation model is formulated in Section II. The statistical model for the MD and map features, the proposed MRMSC-PHD filter, and the proposed novel error metric of the estimated map features are presented in Section III. Section IV presents the experimental and simulation results, which show that our proposed filter outperforms existing MSC-PHD filters by significant margins in terms of average localization and mapping accuracy. Conclusions are given in Section V.

\section{Multipath Modeling}

As shown in Fig. 1, the experimental environment is a typical office area with furniture and cubicles, where the dotted line denotes the trajectory of the MD and the bold lines represent walls, windows, and a large cabinet, which are modeled as planar reflectors. Three RDs are placed at known positions with coordinates $\mathbf{p}_{s}=\left(x_{s}, y_{s}\right)$, where $s=\{1, \ldots, S\}$ and $S=3$ is the number of RDs. 


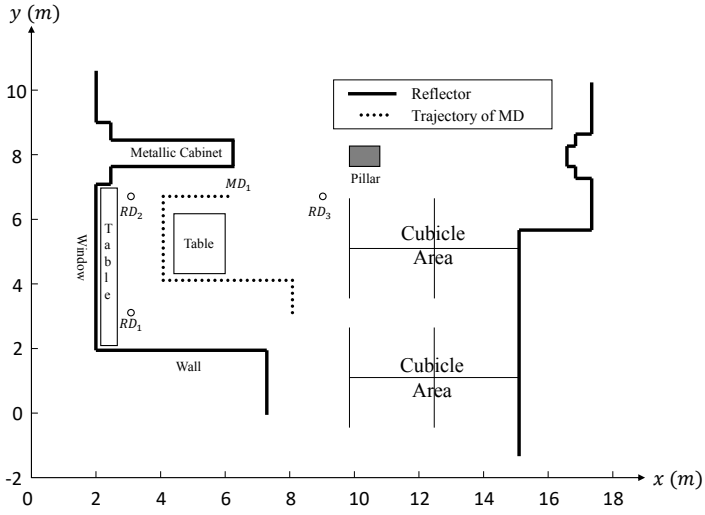

Fig. 1. Environment layout

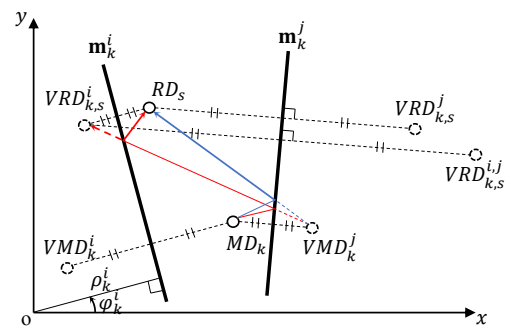

(a)

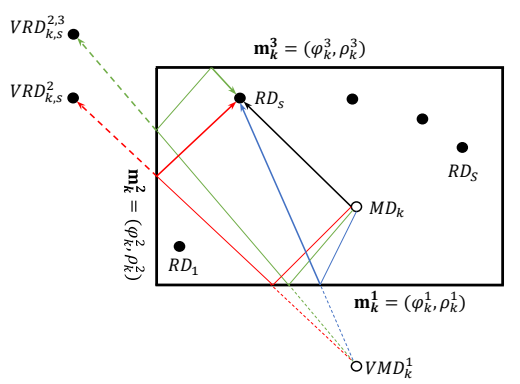

(b)

Fig. 2. Modeling of map features and multipath propagation: (a) Single and double reflection paths and their corresponding VRD and VMD, (b) Illustration of multipath propagation including LOS and multiple reflection paths.

The position of the MD at time $k$ is denoted as $\mathbf{p}_{k}=\left(x_{k}, y_{k}\right)$. Considering reflectors only, the map features can be expressed as $\mathbf{m}=(\varphi, \rho)$. The definition of a planar reflector is based on the Hessian normal form: $x \cos \varphi+y \sin \varphi=\rho$, where $\varphi$ denotes the angle between the planar reflector and the y-axis and $\rho$ represents the distance from the origin to the planar reflector, as shown in Fig. 2(a).

In this paper, all of the higher-order reflection paths are treated as virtual single reflection paths originating from $M D_{k}$ to their corresponding RD or VRD by the following methodology. Fig. 2(a) shows the single and double reflection paths and their corresponding VRD and virtual MD (VMD) [13]. Two map features arise from plane $i$ and plane $j$ at time $k: \mathbf{m}_{k}^{i}=\left(\varphi_{k}^{i}, \rho_{k}^{i}\right)$ and $\mathbf{m}_{k}^{j}=\left(\varphi_{k}^{j}, \rho_{k}^{j}\right)$, respectively. $V R D_{k, s}^{i}$ is the image position of $R D_{s}$ reflected from $\mathbf{m}_{k}^{i}$ at time $k$, which is referred to as a first-order VRD with position $\mathbf{p}_{k, s}^{i}=\left(x_{k, s}^{i}, y_{k, s}^{i}\right) \cdot \mathbf{p}_{k, s}^{i}$ can be calculated using image theory by reflecting $R D_{s}$ through $\mathbf{m}_{k}^{i}$ as

$$
\begin{aligned}
& x_{k, s}^{i}=-x_{s} \cos 2 \varphi_{k}^{i}-y_{s} \sin 2 \varphi_{k}^{i}+2 \rho_{k}^{i} \cos \varphi_{k}^{i} \\
& y_{k, s}^{i}=-x_{s} \sin 2 \varphi_{k}^{i}+y_{s} \cos 2 \varphi_{k}^{i}+2 \rho_{k}^{i} \sin \varphi_{k}^{i}
\end{aligned}
$$

$V R D_{k, s}^{i, j}$ is the image position of $R D_{s}$ reflected from $\left(\mathbf{m}_{k}^{i}, \mathbf{m}_{k}^{j}\right)$ at time $k$, which is referred to as a second-order VRD with position $\mathbf{p}_{k, s}^{i, j}=\left(x_{k, s}^{i, j}, y_{k, s}^{i, j}\right) \cdot \mathbf{p}_{k, s}^{i, j}$ can be calculated using (1) by reflecting $V R D_{k, s}^{i}$ through $\mathbf{m}_{k}^{j}$. Similarly, the third-order VRD of $R D_{s}$ reflected from $\left(\mathbf{m}_{k}^{i}, \mathbf{m}_{k}^{j}, \mathbf{m}_{k}^{l}\right)$ at time $k$ is denoted as $V R D_{k, s}^{i, j, l}$ with position $\mathbf{p}_{k, s}^{i, j, l}=\left(x_{k, s}^{i, j, l}, y_{k, s}^{i, j, l}\right) \cdot \mathbf{p}_{k, s}^{i, j, l}$ can be calculated using (1) by reflecting $V R D_{k, s}^{i, j}$ through $\mathbf{m}_{k}^{l}$. In general, the $m^{\text {th }}$ order VRD of $R D_{s}$ at time $k$ reflected from a vector of the map features $\left(\mathbf{m}_{k}^{i}, \mathbf{m}_{k}^{j}, \mathbf{m}_{k}^{l}, \ldots\right)$, for which the number of elements in the vector is $m$, is denoted as $V R D_{k, s}^{i, j, l, \ldots}$ with position $\mathbf{p}_{k, s}^{i, j, l, \ldots}=\left(x_{k, s}^{i, j, l, \ldots}, y_{k, s}^{i, j, l, \ldots}\right) \cdot \mathbf{p}_{k, s}^{i, j, l, \ldots}$ can be calculated using (1) by reflecting $R D_{s}$ through the vector of the map features $\left(\mathbf{m}_{k}^{i}, \mathbf{m}_{k}^{j}, \mathbf{m}_{k}^{l}, \ldots\right)$ one at a time. Note that the same map feature can appear more than once in the vector of map features. Similarly, the VMD of $M D_{k}$ reflected from $\mathbf{m}_{k}^{j}$ at time $k$ can be denoted as $M D_{k}^{j}$ with position $\mathbf{p}_{k}^{j}=\left(x_{k}^{j}, y_{k}^{j}\right)$, and can be calculated using image theory as

$$
\begin{aligned}
& x_{k}^{j}=-x_{k} \cos 2 \varphi_{k}^{j}-y_{k} \sin 2 \varphi_{k}^{j}+2 \rho_{k}^{j} \cos \varphi_{k}^{j} \\
& y_{k}^{j}=-x_{k} \sin 2 \varphi_{k}^{j}+y_{k} \cos 2 \varphi_{k}^{j}+2 \rho_{k}^{j} \sin \varphi_{k}^{j}
\end{aligned}
$$

Fig. 2(b) shows an example of multipath propagation in a typical indoor environment consisting of an LOS path, a single reflection path, a double reflection path, and a triple reflection path. As shown, the LOS path is independent of the map features and can be used to track the MD, which will help in estimating the map features. All of the reflection paths can be considered virtual single reflection paths originating from $M D_{k}$ reflected from $\mathbf{m}_{k}^{1}$ to their corresponding RD or VRD. For example, the double reflection path originating from $M D_{k}$ reflected from $\left(\mathbf{m}_{k}^{1}, \mathbf{m}_{k}^{2}\right)$ to $R D_{s}$ is considered a virtual single reflection path originating from $M D_{k}$ reflected from $\mathbf{m}_{k}^{1}$ to $V R D_{k, s}^{2}$, which is the first-order VRD of $R D_{s}$ reflected from $\mathbf{m}_{k}^{2}$.

In the remainder of this paper, for ease of presentation, the superscript of VRD that represents the collection of reflected map features is omitted. The collection of $m^{t h}$-order VRDs is denoted as $\left\{V R D_{k, s}^{m, 1}, V R D_{k, s}^{m, 2}, \ldots\right\}$, and its $t^{t h}$ element is denoted as $V R D_{k, s}^{m, t}$.

\section{INDOOR TRACKING WITH MRMSC-PHD FILTER}

In this section, we present the statistical model for the MD and map features and the proposed MRMSC-PHD filter. We show that the LOS-incorporating MSC-PHD (LMSCPHD) filter [25] is a special case of the proposed MRMSCPHD filter. A computationally tractable implementation of the proposed MRMSC-PHD filter is then presented.

\section{A. Statistical Model of MD and map features}

In the two-dimensional (2-D) space, the state vector of the MD can be expressed as $\mathbf{x}_{k}=\left[\mathbf{p}_{k}^{T}, \mathbf{v}_{k}^{T}\right]^{T}$, where $\mathbf{p}_{k}=\left[x_{k}, y_{k}\right]^{T}$ 
and $\mathbf{v}_{k}=\left[\dot{x}_{k}, \dot{y}_{k}\right]^{T}$ represent the position and velocity of the MD at time $k$, respectively. Assuming a linear Gaussian model of constant velocity, the dynamical model of the MD can be expressed as

$$
\begin{aligned}
\mathbf{x}_{k} & =\mathbf{F} \mathbf{x}_{k-1}+\mathbf{G n}_{k-1, a} \\
& =\left[\begin{array}{cc}
\mathbf{I}_{2} & \Delta T \cdot \mathbf{I}_{2} \\
\mathbf{0}_{2} & \mathbf{I}_{2}
\end{array}\right] \mathbf{x}_{k-1}+\left[\begin{array}{c}
\frac{\Delta T^{2}}{2} \cdot \mathbf{I}_{2} \\
\Delta T \cdot \mathbf{I}_{2}
\end{array}\right] \mathbf{n}_{k-1, a}
\end{aligned}
$$

where $\mathbf{n}_{k-1, a}$ is the driving acceleration noise with zero mean and covariance matrix $\sigma_{a}^{2} \mathbf{I}_{2}$, and $\sigma_{a}$ is the acceleration noise in either direction. $\mathbf{I}_{N}$ and $\mathbf{0}_{N}$ represent the $N \times N$ identity and zero matrix, respectively. $\Delta T$ denotes the sampling interval.

Because the number of detected map features at time $k$, denoted as $\left|M_{k}\right|$, is time-variant as the MD moves around, the collection of detected map features can be modeled as an RFS [29]. The $i^{t h}$ map feature at time $k$ is denoted as $\mathbf{m}_{k}^{i}=$ $\left[\varphi_{k}^{i}, \rho_{k}^{i}\right]^{T}$. Assuming that all of the map features are static, the dynamical model of the map features can be expressed as

$$
M_{k}=M_{k-1}=\left\{\mathbf{m}_{k}^{1}, \ldots, \mathbf{m}_{k}^{\left|M_{k}\right|}\right\}
$$

where $M_{k-1}$ represents the map features estimated at time $k-1$.

Given the $R D_{s}$ with position $\mathbf{p}_{s}=\left(x_{s}, y_{s}\right)$ and $M D_{k}$ with position $\mathbf{p}_{k}=\left(x_{k}, y_{k}\right)$, the LOS TOA measured by $R D_{s}$ at time $k$ can be defined as

$$
z_{k, s}^{l}=\mathbf{h}_{s}^{l}\left(\mathbf{p}_{k}, \mathbf{p}_{s}\right)+n_{k, s}^{l}=\sqrt{\left(x_{k}-x_{s}\right)^{2}+\left(y_{k}-y_{s}\right)^{2}}+n_{k, s}^{l}
$$

where $n_{k, s}^{l}$ represents the Gaussian measurement noise of the LOS path with zero mean and standard deviation of $\sigma_{z}^{l}$.

The measured TOA of the reflection paths can be calculated as follows. The $m^{t h}$-order reflection paths generated by the $j^{t h}$ map feature $\mathbf{m}_{k}^{j}$ to $V R D_{k, s}^{m-1, t}$ can be expressed as

$$
z_{k, s}^{m, t}=\mathbf{h}_{s}^{m, t}\left(\mathbf{p}_{k}, \mathbf{m}_{k}^{j}, \mathbf{p}_{k, s}^{m-1, t}\right)+n_{k, s}^{m}=\mathbf{h}_{s}^{l}\left(\mathbf{p}_{k}^{j}, \mathbf{p}_{k, s}^{m-1, t}\right)+n_{k, s}^{m}
$$

where $n_{k, s}^{m}$ represents the Gaussian measurement noise of the $m^{\text {th }}$-order reflections with zero mean and standard deviation of $\sigma_{z}^{m} \cdot \mathbf{p}_{k}^{j}=\left(x_{k}^{j}, y_{k}^{j}\right)$ denotes the VMD position obtained by reflecting the MD through $\mathbf{m}_{k}^{j}$, which can be calculated according to image theory as in (2). $\mathbf{p}_{k, s}^{m-1, t}$ is the position of $V R D_{k, s}^{m-1, t}$, which can be calculated using image theory as in (1).

The number of measurements received at each RD depends on the detected map features and the clutter, the latter of which is time-variant. Therefore, the collection of measurements should be modeled as an RFS:

$$
Z_{k, s}=\mathcal{D}\left(\mathbf{x}_{k}\right) \cup \mathcal{D}\left(\mathbf{x}_{k}, M_{k, s}\right) \cup C_{k, s} \in \mathcal{F}(\mathcal{Z})
$$

where $\mathcal{F}(\mathcal{Z})$ denotes the collection of all of the finite subsets of $\mathcal{Z} . \mathcal{D}\left(\mathbf{x}_{k}\right)$ is the collection of measurements from the LOS path whose elements are given in (5). $\mathcal{D}\left(\mathbf{x}_{k}, M_{k, s}\right)$ is the collection of measurements from those reflections whose elements are shown in (6). $C_{k, s}$ is an RFS denoting the clutter received by $R D_{s}$, such as diffractions, which can be formulated as a Poisson point process with intensity $c_{s}(z)=\lambda_{c}^{s} U(z)$, where $\lambda_{c}^{s}$ is the clutter rate, which indicates the average amount of clutter measured by $R D_{s}$ [30]. $U(\cdot)$ represents a uniform density over the maximum detected distance. Then, the joint measurement set obtained by all of the RDs at time $k$ can be expressed as $Z_{k}=Z_{k, 1} \cup \cdots \cup Z_{k, S}$.

The prediction and updating for the joint probability density of the MD and map can thus be written as [26]

$$
\begin{array}{r}
p_{k \mid k-1}\left(\mathbf{x}_{k}, M_{k} \mid Z_{1: k-1}\right)=\iint f_{k \mid k-1}\left(\mathbf{x}_{k}, M_{k} \mid \mathbf{x}_{k-1}, M_{k-1}\right) \\
\times p_{k-1}\left(\mathbf{x}_{k-1}, M_{k-1} \mid Z_{1: k-1}\right) \mathrm{d} \mathbf{x}_{k-1} \delta M_{k-1} \\
p_{k \mid k}\left(\mathbf{x}_{k}, M_{k} \mid Z_{1: k}\right) \\
=\frac{p_{k \mid k-1}\left(\mathbf{x}_{k}, M_{k} \mid Z_{1: k-1}\right) L_{k}\left(Z_{k} \mid \mathbf{x}_{k}, M_{k}\right)}{\iint p_{k \mid k-1}\left(\mathbf{x}_{k}, M_{k} \mid Z_{1: k-1}\right) L_{k}\left(Z_{k} \mid \mathbf{x}_{k}, M_{k}\right) \mathrm{d} \mathbf{x}_{k} \delta M_{k}}
\end{array}
$$

where $f_{k \mid k-1}\left(\mathbf{x}_{k}, M_{k} \mid \mathbf{x}_{k-1}, M_{k-1}\right)$ and $L_{k}\left(Z_{k} \mid \mathbf{x}_{k}, M_{k}\right)$ are the joint Markov transition density and measurement likelihood at time $k$, respectively. $p_{k-1}\left(\mathbf{x}_{k-1}, M_{k-1} \mid Z_{1: k-1}\right)$ is the joint posterior density of the MD and map at time $k-1$. Note that the integrals of the map features are set integrals, which are computationally intractable [29].

\section{B. Likelihood Model}

As stated earlier, the measurements consist of the LOS path, reflections, and clutter. For any nonempty measurement subset $W \in Z_{k}$, given the MD state $\mathbf{x}_{k}$ and map feature $\mathbf{m}_{k}^{j}$, the multiobject likelihood function can be calculated as follows. If the subset $W$ represents the LOS measurements, the multi-object likelihood function can be expressed as

$$
L_{W}^{l}\left(\mathbf{x}_{k}\right)= \begin{cases}\left(1-\tilde{P}_{d}^{l, s}\right) \prod_{s<W} P_{d}^{l, s} l_{z_{s}}^{l, s}, & \text { if } W_{s}=\left\{z_{s}\right\} \\ 0, & \text { otherwise }\end{cases}
$$

where $1-\tilde{P}_{d}^{l, s}=\prod_{s \nless W}\left(1-P_{d}^{l, s}\right) . s<W$ and $s \nless W$ mean that the $W$ does and does not contain measurements from $R D_{s}$, respectively. $P_{d}^{l, s}$ and $l_{z}^{l, s}$ are abbreviations of $P_{d}^{l, s}\left(\mathbf{x}_{k}\right)$ and $l_{z}^{l, s}\left(\mathbf{x}_{k}\right)$, which represent the LOS detection probability and the measurement likelihood function of $R D_{s}$, respectively. $W_{s}$ denotes the collection of elements in $W$ from $R D_{s}$. According to (5), the LOS measurement likelihood can be modeled as $\mathcal{N}\left(z ; \mathbf{h}_{s}^{l}\left(\mathbf{p}_{k}, \mathbf{p}_{s}\right),\left(\sigma_{z}^{l}\right)^{2}\right)$, where $\mathcal{N}(\cdot ; \mu, \Sigma)$ represents a Gaussian distribution with mean $\mu$ and covariance $\Sigma$.

If the subset $W$ represents reflection measurements, the multi-object likelihood function can be expressed as

$$
\begin{aligned}
& L_{W}^{r}\left(\mathbf{m}_{k}^{j} \mid \mathbf{x}_{k}\right)=\left(1-\tilde{P}_{d}^{r, s}\right) \\
& \quad \times \prod_{s<W}\left(\sum_{\theta_{s}}\left(\prod_{\theta_{s}(m, t)=0}\left(1-P_{d}^{m, s, t}\right) \prod_{\theta_{s}(m, t)>0} P_{d}^{m, s, t} l_{z_{\theta_{s}(m, t)}^{m, s, t}}\right)\right.
\end{aligned}
$$

where $1-\tilde{P}_{d}^{r, s}=\prod_{s \nless W} \prod_{m} \prod_{t=1}^{n_{k}^{m}}\left(1-P_{d}^{m, s, t}\right)$, where $n_{k}^{m}$ represents the number of $m^{t h}$-order reflection paths. $\theta_{s}$ represents all of the possible associations between the reflection paths from $R D_{s}$ and the elements in $W_{s} . \theta_{s}(m, t)=0$ indicates that the path traced to $V R D_{k, s}^{m-1, t}$ is not detected, while $\theta_{s}(m, t)>0$ indicates that the path traced to $V R D_{k, s}^{m-1, t}$ is detected and is associated with the element $z_{\theta_{s}(m, t)}$ in $W_{s} . P_{d}^{m, s, t}$ and $l_{z}^{m, s, t}$ are abbreviations of $P_{d}^{m, s, t}\left(\mathbf{m} \mid \mathbf{x}_{k}, \mathbf{p}_{k, s}^{m-1, t}\right)$ and $l_{z}^{m, s, t}\left(\mathbf{m} \mid \mathbf{x}_{k}, \mathbf{p}_{k, s}^{m-1, t}\right)$, which represent the detection probability and measurement 


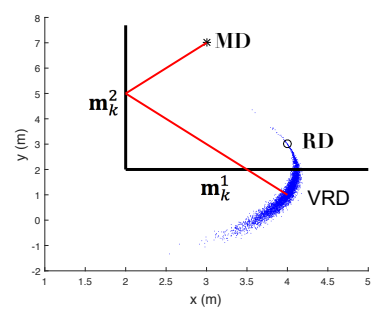

(a)

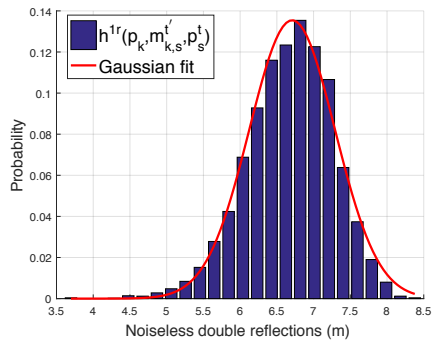

(b)
Fig. 3. Gaussian fit of a double reflection between $M D$ and RD via two map features with $\sigma_{\varphi}=5^{\circ}$ and $\sigma_{\rho}=0.1 \mathrm{~m}$ : (a) Calculated VRD position corresponding to 5000 realizations of $\mathbf{m}_{2}$, (b) Gaussian fit of noiseless double reflections.

likelihood of the $m^{t h}$-order reflection path traced to $V R D_{k, s}^{m-1, t}$ with position $\mathbf{p}_{k, s}^{m-1, t}$. According to (5), the measurement likelihood of a single reflection conditional on a given map feature $\mathbf{m}_{k}^{j}$ can be modeled as $\mathcal{N}\left(z ; \mathbf{h}_{s}^{l}\left(\mathbf{p}_{k}^{j}, \mathbf{p}_{s}\right),\left(\sigma_{z}^{1}\right)^{2}\right)$. However, the modeling of higher-order reflections is more complex because it involves uncertainties regarding the VRD position, as shown in (6).

In this paper, we assume that the map features $\mathbf{m}_{k}$ are Gaussian-distributed random variables. Further, we assume that $\mathbf{h}_{s}^{l}\left(\mathbf{p}_{k}^{j}, \mathbf{p}_{k, s}^{m-1, t}\right)$ also follows a Gaussian distribution for ease of implementation, and we use a numerical method to find its mean and standard deviation. Fig. 3 gives an example of the use of a Gaussian distribution to fit the likelihood of double reflections. As shown in Fig. 3(a), there are two map features with mean positions of $\overline{\mathbf{m}}_{k}^{1}=(\pi / 2,2)$ and $\overline{\mathbf{m}}_{k}^{2}=(0,2)$ and the same standard deviation $\sigma_{\varphi}=5^{\circ}$ and $\sigma_{\rho}=0.1 \mathrm{~m}$. The calculated VRDs corresponding to 5000 realizations of $\mathbf{m}_{k}^{1}$ are also shown. The noiseless path length of double reflections for each generated VRD can be calculated and fitted using a Gaussian distribution as shown in Fig. 3(b). The mean and standard deviation of the Gaussian distribution can be denoted as $\mu_{k, s}^{m}=\mathbf{h}_{s}^{l}\left(\mathbf{p}_{k}^{2}, \overline{\mathbf{p}}_{k, s}^{m-1,1}\right)$ and $\sigma_{k, s}^{m}$, respectively, where $m=2$ and $\overline{\mathbf{p}}_{k, s}^{m-1,1}$ means the VRD position calculated using $\overline{\mathbf{m}}_{k}^{1}$. The $\sigma_{k, s}^{m}$ should be estimated numerically. As can be seen, the noiseless double reflections fit quite well. The likelihood functions of the higher-order reflections can be estimated similarly. Note that $\sigma_{k, s}^{m}$ must be numerically estimated for each particle, each reflection path, and each time $k$ because it depends on the relative positions of the MD and VRD. After estimating the standard deviation $\sigma_{k, s}^{m}, l_{z}^{m, s, t}$ can be approximated using $\mathcal{N}\left(z ; \mu_{k, s}^{m},\left(\sigma_{k, s}^{m}\right)^{2}+\left(\sigma_{z}^{m}\right)^{2}\right)$.

If the subset $W$ represents clutter, the multi-object likelihood function can be expressed as

$$
c_{W}= \begin{cases}c_{s}\left(z_{s}\right), & \text { if } W=\left\{z_{s}\right\} \\ 0, & \text { otherwise. }\end{cases}
$$

$c_{S}(z)$ indicates the intensity of clutter as defined in (7).

\section{Assumptions}

Before we present the derived MRMSC-PHD filter, we first summarize the assumptions made in the conventional MSCPHD filter [28], [40]: (1) The map features are modeled as a Poisson point process whose state is conditional on the MD position, which is also a stochastic process; (2) There is no LOS path; (3) Each map feature generates at most one measurement, which is a single reflection in the indoor scenario; (4) Measurements generated by the map features are conditionally independent of each other given the MD position; and (5) The clutter measurement is modeled as a Poisson process and is independent of measurements generated by map features. For the indoor MRMSC-PHD filter, assumptions (1), (4), and (5) are kept, while assumption (2) is removed so that the LOS path is either detected or blocked. More importantly, assumption (3) is relaxed such that each map feature can generate more than one measurement through multiple reflections.

\section{Proposed MRMSC-PHD Filter}

Based on the assumption of a static map, the joint prediction of the MD and map features can be expressed as [25]

$$
\begin{aligned}
v_{k \mid k-1}\left(\mathbf{x}_{k}, \mathbf{m}\right)=\int & f_{k \mid k-1}\left(\mathbf{x}_{k} \mid \mathbf{x}_{k-1}\right) p_{k-1}\left(\mathbf{x}_{k-1} \mid Z_{1: k-1}\right) \\
& \times v_{k-1}\left(\mathbf{m} \mid \mathbf{x}_{k-1}\right) \mathrm{d} \mathbf{x}_{k-1}
\end{aligned}
$$

where $f_{k \mid k-1}\left(\mathbf{x}_{k} \mid \mathbf{x}_{k-1}\right)$ and $p_{k-1}\left(\mathbf{x}_{k-1} \mid Z_{1: k-1}\right)$ represent the marginal Markov transition density at time $k$ and the posterior distribution of the MD at time $k-1$, respectively. $v_{k-1}\left(\mathbf{m} \mid \mathbf{x}_{k-1}\right)$ represents the conditional posterior PHD of map features at time $k-1$.

The joint updating of the MRMSC-PHD filter can be derived as

$$
\begin{aligned}
& v_{k}\left(\mathbf{x}_{k}, \mathbf{m}\right)=\sum_{\mathcal{P}_{\sqsubset} Z_{k}} \omega_{\mathcal{P}} \frac{p_{k \mid k-1}\left(\mathbf{x}_{k} \mid Z_{1: k-1}\right) L_{\mathcal{P}}\left(\mathbf{x}_{k}\right)}{p_{k \mid k-1}\left[L_{\mathcal{P}}\right]} v_{k}\left(\mathbf{m} \mid \mathbf{x}_{k}\right) \\
& v_{k}\left(\mathbf{m} \mid \mathbf{x}_{k}\right)=\left(1-\tilde{P}_{d}^{r}+\sum_{W \in \mathcal{P}}\left(1-\Omega_{W}\right) \frac{L_{W}^{r}}{\Gamma_{W}}\right) v_{k \mid k-1}\left(\mathbf{m} \mid \mathbf{x}_{k}\right)
\end{aligned}
$$

where

$$
\begin{gathered}
\Gamma_{W}=c_{W}+v_{k \mid k-1}\left[L_{W}^{r} \mid \mathbf{x}_{k}\right], \omega \mathcal{P}=\frac{p_{k \mid k-1}\left[L_{\mathcal{P}}\right]}{\sum_{Q \sqsubset Z_{k}} p_{k \mid k-1}\left[L_{Q}\right]} \\
L_{\mathcal{P}}\left(\mathbf{x}_{k}\right)=\frac{\left(1-\tilde{P}_{d}^{l}+\sum_{W \in \mathcal{P}}\left(L_{W}^{l} / \Gamma_{W}\right)\right) \prod_{W \in \mathcal{P}} \Gamma_{W}}{\exp ^{v_{k \mid k-1}\left[\tilde{P}_{d}^{r} \mid \mathbf{x}_{k}\right]}} \\
\Omega_{W}=\frac{L_{W}^{l} / \Gamma_{W}}{1-\tilde{P}_{d}^{l}+\sum_{W \in \mathcal{P}}\left(L_{W}^{l} / \Gamma_{W}\right)}
\end{gathered}
$$

$p_{k \mid k-1}\left(\mathbf{x}_{k} \mid Z_{1: k-1}\right)$ and $v_{k}\left(\mathbf{m} \mid \mathbf{x}_{k}\right)$ in (12) denote the predicted marginal distribution of the MD and the updated conditional PHD of the map features at time $k$, respectively. $\omega \mathcal{P}$ and $L \mathcal{P}\left(\mathbf{x}_{k}\right)$ in (12) represent the weight and the multi-RD multiobject measurement likelihood of partition $\mathcal{P}$, respectively. $\Omega_{W}$ in (12) represents the coefficient of the LOS path. When updating map features, $\Omega_{W}$ is subtracted, because the LOS path is independent of map features. Note that the term $\Omega_{W}$ distinguishes the proposed updating formula from the formula in [33]. $L_{W}^{l}, L_{W}^{r}, c_{W}$ are likelihood functions defined in (8), (9), (10), respectively. $1-\tilde{P}_{d}^{l}=\prod_{s}\left(1-P_{d}^{l, s}\right)$ and $1-\tilde{P}_{d}^{r}=\prod_{s} \prod_{m} \prod_{t=1}^{n_{k}^{m}}\left(1-P_{d}^{m, s, t}\right) . \mathcal{P} \sqsubset Z_{k}$ represents the whole 


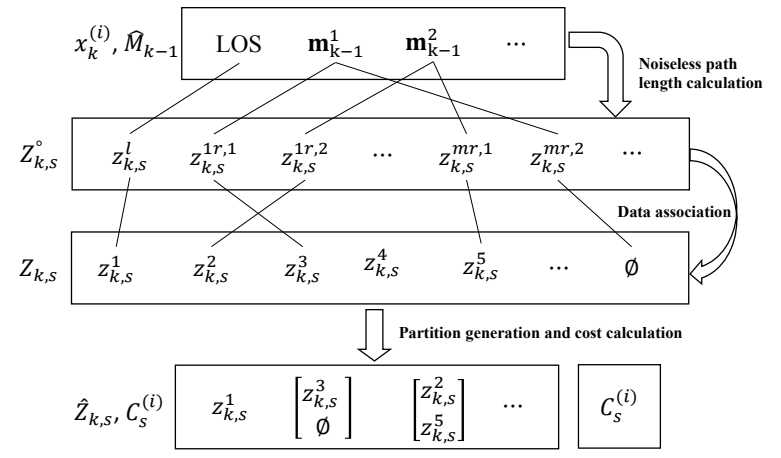

Fig. 4. Data association between measurements $Z_{k, s}$ and theoretical path length $Z_{k, s}^{\circ}$

partition of the measurement set $Z_{k}$. The detailed derivation of (12) is shown in Appendix A.

When $P_{d}^{m, s, t}=0$ for all double and higher reflections, which means that there are only single reflections, the MRMSC-PHD filter simplifies to the LMSC-PHD filter proposed in [25]. The reflections' related terms $\tilde{P}_{d}^{r}$ and $L_{W}^{r}$ are reduced to

$$
\tilde{P}_{d}^{r}=1-\prod_{s}\left(1-P_{d}^{1, s}\right), L_{W}^{r}=\prod_{s \nless W}\left(1-P_{d}^{1, s}\right) \prod_{s<W} P_{d}^{1, s} l_{z_{s}}^{1, s}
$$

The expressions of the other terms remain the same, and the resulting formulas denote the explicit form of the LMSC-PHD filter. If it is further assumed that $P_{d}^{l, s}=0$ in the LMSC-PHD filter, which means that there is no LOS path, then the filter simplifies to the conventional MSC-PHD filter [26].

\section{E. Implementation of MRMSC-PHD Filter}

The proposed MRMSC-PHD filter is implemented using a particle filter to model the MD and a Gaussian-mixture (GM) filter to characterize the map features. The predictive method can be found in [25]. Assume that the predicted densities of the MD and PHD of the map features at time $k$ are

$$
\begin{array}{r}
p_{k \mid k-1}\left(\mathbf{x} \mid Z_{1: k-1}\right)=\sum_{l=1}^{N_{k \mid k-1}} \omega_{k \mid k-1}^{(l)} \delta\left(\mathbf{x}-\mathbf{x}_{k}^{(l)}\right) \\
v_{k \mid k-1}\left(\mathbf{m} \mid \mathbf{x}_{k}^{(l)}\right)=\sum_{J=1}^{J_{k \mid k-1}^{(l)}} \eta_{k \mid k-1}^{(J, l)} \mathcal{N}\left(\mathbf{m} ; \overline{\mathbf{m}}_{k \mid k-1}^{(J, l)}, \mathbf{P}_{k \mid k-1}^{(J, l)}\right)
\end{array}
$$

where $\delta(\cdot)$ is the Dirac function. $\omega_{k \mid k-1}^{(l)}$ and $\eta_{k \mid k-1}^{(J, l)}$ are the corresponding weights of the particle and GM components, respectively. $N_{k \mid k-1}$ and $J_{k \mid k-1}^{(l)}$ are the number of particles and number of GM components of the $t^{\text {th }}$ particle, respectively.

1) Greedy Partition Based on the OSPA Metric: According to (12), when updating the MD and map features, all possible partitions of the measurements should be considered. To avoid the combinatorial increase of the computation load, a greedy partition method based on the optimal subpattern assignment (OSPA) metric is presented. The OSPA metric is proposed in [41] and is used to define the difference between the two sets. For the two sets $X$ and $Y$ with $|X|<=|Y|$, the OSPA distance $\bar{d}_{p}^{(c)}(X, Y)$ between $X$ and $Y$ with some $c>0$ and $1 \leq p<\infty$ is defined as

$\bar{d}_{p}^{(c)}(X, Y)=\left(\frac{1}{|Y|}\left(\min _{\pi \in \Pi_{|Y|}} \sum_{i=1}^{|X|} d^{(c)}\left(x_{i}, y_{\pi(i)}\right)^{p}+c^{p}(|X|-|Y|)\right)\right)^{1 / p}$

where $d^{(c)}(x, y)=\min (c, d(x, y))$ denotes an arbitrary distance between $x$ and $y$ cut off at $c . \Pi_{k}$ represents the set of permutations of $\{1,2, \ldots, k\}$ for any $k \in \mathbb{N}$.

For each particle $\mathbf{x}_{k}^{(l)}$ at time $k$, a set of noiseless path lengths $Z_{k, s}^{\circ}$ including the LOS path and reflections to $R D_{s}$ can be calculated based on the estimated map $\hat{M}_{k-1}$. Then, for each particle $\mathbf{x}_{k}^{(l)}$, we can obtain a set of the closest measurements $\hat{Z}_{k, s}$ and the corresponding cost function $C_{s}^{(l)}=\bar{d}_{1}^{(c)}\left(Z_{k, s}^{\circ}, Z_{k, s}\right)$ by associating the measurement set $Z_{k, s}$ with $Z_{k, s}^{\circ}$, as shown in Fig. 4. As can be seen, the path associated with $\emptyset$ indicates that the corresponding path is not detected. If the measurement is not associated with any path, it is considered clutter. After estimating $\hat{Z}_{k, s}$ for each RD, the measurements generated by the LOS path and each map feature can be concatenated. Then, the partition based on the current particle and corresponding cost function $C^{(l)}=\sum_{s} C_{s}^{(l)}$ can be constructed. After calculating the $\operatorname{cost} C^{(l)}$ of all of the particles, the $N_{\max }^{P}$ partitions with minimum $C^{(l)}$ are selected as possible partitions.

2) Implementation of Update: After performing the greedy partition, the MD and map features based on each partition can be updated as follows:

$$
v_{k}\left(\mathbf{m} \mid \mathbf{x}_{k}^{(l)}\right)=v_{k}^{m d}\left(\mathbf{m} \mid \mathbf{x}_{k}^{(l)}\right)+\sum_{W \in \mathcal{P}} v_{k}^{d}\left(\mathbf{m}, W \mid \mathbf{x}_{k}^{(l)}\right)
$$

where $v_{k}^{m d}$ and $v_{k}^{d}$ represent the mis-detected and detected parts of the map features at time $k$, respectively, which can be expressed as

$$
\begin{gathered}
v_{k}^{m d}\left(\mathbf{m} \mid \mathbf{x}_{k}^{(l)}\right)=\sum_{J=1}^{J_{k-1}^{(l)}}\left(1-\tilde{P}_{d}^{r}\right) \eta_{k-1}^{(J, l)} \mathcal{N}\left(\mathbf{m} ; \overline{\mathbf{m}}_{k-1}^{(J, l)}, \mathbf{P}_{k-1}^{(J, l)}\right) \\
v_{k}^{d}\left(\mathbf{m}, W \mid \mathbf{x}_{k}^{(l)}\right)=\sum_{J=1}^{J_{k-1}^{(l)}} \eta_{k}^{(J, l)} \mathcal{N}\left(\mathbf{m} ; \overline{\mathbf{m}}_{k}^{(J, l)}, \mathbf{P}_{k}^{(J, l)}\right)
\end{gathered}
$$

The weight of each updated map feature using subset $W$ can be expressed as

$$
\eta_{k}^{(J, l)}=\left(1-\Omega_{W}^{(l)}\right) \eta_{k-1}^{(J, l)} q_{W}^{(J, l)} / \Gamma_{W}^{(l)}
$$

where

$$
\begin{gathered}
\Omega_{W}^{(l)}=\frac{L_{W}^{l} / \Gamma_{W}^{(l)}}{1-\tilde{P}_{d}^{l}+\sum_{W \in \mathcal{P}}\left(L_{W}^{l} / \Gamma_{W}^{(l)}\right)}, \Gamma_{W}^{(l)}=c_{W}+\sum_{J} \eta_{k-1}^{(J, l)} \phi_{W}^{(J, l)} \\
\phi_{W}^{(J, l)}=\left(1-\tilde{P}_{d}^{r, s}\right) \prod_{s<W}\left(\sum _ { \theta _ { s } } \left(\prod_{\theta_{s}(m, t)=0}\left(1-P_{d}^{m, s, t}\right)\right.\right. \\
\left.\left.\times \prod_{\theta_{s}(m, t)>0} P_{d}^{m, s, t} q_{W}^{(J, l)}\right)\right) \\
q_{W}^{(J, l)}=\mathcal{N}\left(\mathbf{z}_{W}, \mathbf{y}_{W}, \mathbf{H}_{W} \mathbf{P}_{k \mid k-1}^{(J, l)} \mathbf{H}_{W}^{T}+\mathbf{R}_{W}\right)
\end{gathered}
$$




$$
\begin{gathered}
\mathbf{z}_{W}=\bigoplus_{z \in W} z, \mathbf{y}_{W}=\bigoplus_{\theta_{s}(m, t)>0} \mathbf{h}_{s}^{m, t}\left(\mathbf{x}_{k}^{l}, \overline{\mathbf{m}}_{k-1}^{(J, l)}, \mathbf{p}_{k, s}^{m-1, t}\right) \\
\mathbf{H}_{W}=\left.\bigoplus_{\theta_{s}(m, t)>0} \frac{\partial \mathbf{h}_{s}^{m, t}\left(\mathbf{x}_{k}^{l}, \mathbf{m}_{k}^{j}, \mathbf{p}_{k, s}^{m-1, t}\right)}{\partial \mathbf{m}_{k}^{j}}\right|_{\mathbf{m}_{k}^{j}=\overline{\mathbf{m}}_{k-1}^{(J, l)}}
\end{gathered}
$$

$\mathbf{R}_{W}$ is a diagonal covariance matrix with diagonal elements equal to $\left(\sigma_{z}^{1}\right)^{2}$ for single reflections and $\left(\sigma^{m}\right)^{2}+\left(\sigma_{z}^{m}\right)^{2}$ for higher-order reflections. $\bigoplus$ means vertical vectorial concatenation. The Gaussian components in (17) are

$$
\begin{gathered}
\overline{\mathbf{m}}_{k \mid k}^{(J, l)}=\overline{\mathbf{m}}_{k \mid k-1}^{(J, l)}+\mathbf{K}_{k}\left[\mathbf{z}_{\mathbf{W}}-\mathbf{y}_{W}\right] \\
\mathbf{K}_{k}=\mathbf{P}_{k \mid k-1}^{(J, l)} \mathbf{H}_{W}^{T}\left(\mathbf{H}_{W} \mathbf{P}_{k \mid k-1}^{(J, l)} \mathbf{H}_{W}^{T}+\mathbf{R}_{W}\right)^{-1} \\
\mathbf{P}_{k \mid k}^{(J, l)}=\left[\mathbf{I}-\mathbf{K}_{k} \mathbf{H}_{W}\right] \mathbf{P}_{k \mid k-1}^{(J, l)}
\end{gathered}
$$

Then $L_{\mathcal{P}}\left(\mathbf{x}_{\mathbf{k}}\right), \omega_{\mathcal{P}}$ and $\omega_{k \mid k}^{(l)}$ can be calculated as

$$
\begin{gathered}
L_{\mathcal{P}}^{(l)}=\frac{\left(1-\tilde{P}_{d}^{l}+\sum_{W \in \mathcal{P}}\left(L_{W}^{l} / \Gamma_{W}^{(l)}\right)\right)}{\exp _{d}^{\tilde{P}_{d}^{r} \sum_{j} \eta_{k \mid k-1}^{(J, l)}}} \prod_{W \in \mathcal{P}} \Gamma_{W}^{(l)} \\
\omega_{\mathcal{P}}=\frac{\sum_{l} L_{\mathcal{P}}^{(l)} \omega_{k \mid k-1}^{(l)}}{\sum_{\mathcal{} \sqsubset Z_{k}} \sum_{l} L_{\mathcal{Q}}^{(l)} \omega_{k \mid k-1}^{(l)}}, \omega_{k \mid k}^{(l)}=\frac{\sum_{\mathcal{P}} \omega_{\mathcal{P}} L_{\mathcal{P}}^{(l)} \omega_{k \mid k-1}^{(l)}}{\sum_{l} L_{\mathcal{P}}^{(l)} \omega_{k \mid k-1}^{(l)}}
\end{gathered}
$$

Finally, the state of the MD is estimated using the weighted average, and the corresponding posterior PHD of the map features can be estimated using the weighted average of the trajectory-conditioned PHD, which can be expressed as

$$
\hat{\mathbf{x}}_{k}=\frac{\sum_{l} \omega_{k \mid k}^{(l)} \mathbf{x}_{k}^{(l)}}{\sum_{i} \omega_{k \mid k}^{(l)}}, v_{k \mid k}\left(\mathbf{m} \mid \mathbf{x}_{k}\right)=\frac{\sum_{l} \omega_{k \mid k}^{(l)} v_{k \mid k}\left(\mathbf{m} \mid \mathbf{x}_{k}^{(l)}\right)}{\sum_{l} \omega_{k \mid k}^{(l)}}
$$

Then, the expected map features are estimated as the local maxima of the posterior PHD $v_{k \mid k}\left(\mathbf{m} \mid \mathbf{x}_{k}\right)$ with weight greater than a threshold, which is 0.5 in this paper [30]. Note that the number of GM terms grows exponentially during the recursion, so pruning should be performed after each update to retain the most $N_{\max }^{G M}$ GM components of each particle [30].

\section{F. Identifiability}

In [20], the identifiability of the joint MD and multipath parameter estimation with both TOA and angle-of-arrival (AOA) measurements are analyzed by studying the Cramer-Rao lower bound (CRLB) for the estimated parameters. Ref. [42] also discusses the identifiability of a network localization and mapping, but one based only on TOA measurements. By using at least three LOS paths, the position of an MD can be unambiguously localized. Likewise, if at least three single reflection paths are reflected from the same map feature, the feature can be unambiguously estimated [42]. If each map feature satisfies this condition and the correct data association is known, then the whole map can be constructed unambiguously. Then, with the unambiguously estimated map and the correct data association known, the MD can be localized using three paths, which can be either the LOS or reflections. Although the correct data association is usually unknown, it can still be estimated by utilizing the prior position information of the MD. In the simulation, after the MD and map features are unambiguously initialized, the MD will remain tracked by at least three paths (either LOS or reflection paths).

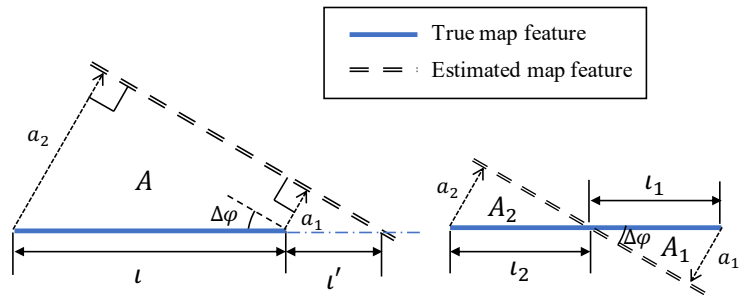

(a)

(b)

Fig. 5. Proposed error metric of the estimated map feature: (a) A case in which the estimated map feature does not cross the true map feature and (b) a case in which the estimated map feature crosses the true map feature.

\section{G. Complexity Analysis}

Suppose that the number of particles is $N_{p}$, the maximum number of GM components of each particle is $N_{\max }^{G M}$, the number of maximum partitions is $N_{\text {max }}^{P}$, the number of $m^{t h}$ order reflection paths is $n^{m}$ where $m$ is the order of reflection, and the number of RDs is $S$. Then, the complexity of the predictive calculations using the proposed MRMSCPHD filter is $O\left(N_{p} N_{\max }^{G M}\right)$, the complexity of the proposed greedy partition algorithm is $O\left(N_{p} S\left(1+\sum_{m} n^{m}\right)\right)$, and the complexity of the update calculations the proposed MRMSCPHD filter is $O\left(N_{p} N_{\max }^{P} N_{\max }^{G M}(1+m)\right)$. Therefore, the total complexity of the proposed filter can be considered to be $O\left(N_{p} N_{\max }^{P} N_{\max }^{G M}(1+m)\right)$, which is linear with the number of reflections.

\section{H. Error Metric}

After estimating the MD and map features, it is important to evaluate the performance of the proposed MRMSC-PHD filter. For the MD position $\mathbf{p}_{k}$ at time $k$, the localization error can be calculated as $\left|\mathbf{p}_{k}-\hat{\mathbf{p}}_{k}\right|$, where $\hat{\mathbf{p}}_{k}$ denotes the estimated position of the MD and $|\cdot|$ represents the 2-norm. For the collection of map features $M_{k}$ at time $k$, the mapping error is defined using the OSPA metric $\bar{d}_{1}^{(c)}\left(M_{k}, \hat{M}_{k}\right)$ as shown in (15), where $\hat{M}_{k}$ is the collection of estimated map features. However, to calculate the error metric of the estimated map features $d_{1}^{(c)}(\mathbf{m}, \hat{\mathbf{m}})$, it should be defined as in (15). For an outdoor radar-based scenario, where map features are treated as points, the error metric $d_{1}^{(c)}(\mathbf{m}, \hat{\mathbf{m}})$ can be defined using Euclidean distance [23], [26]. However, for an indoor environment, where map features are treated as planar reflectors, using Euclidean distance to define the error in $\varphi-\rho$ space results in two issues. First, $\varphi$ and $\rho$ involve different units. Second, the Euclidean distance is dependent on the choice of origin.

Therefore, we propose a new error metric $d_{1}^{(c)}(\mathbf{m}, \hat{\mathbf{m}})$ that provides the average distance between an estimated planar map feature and the true planar map feature, as shown in Fig. 5. Suppose that the distances of two perpendicular lines from the two end points of the true map feature to the estimated map feature are $a_{1}$ and $a_{2}$, and the resulting enclosed area for case (a) is $A$ and that for case (b) is $A_{1}+A_{2}$; the error metric can 
then be defined as

$$
d_{1}^{(c)}(\mathbf{m}, \hat{\mathbf{m}})=\left\{\begin{array}{ll}
\frac{A}{\iota}, \\
\frac{A_{1}+A_{2}}{\iota_{1}+\iota_{2}},
\end{array}= \begin{cases}\frac{a_{1}+a_{2}}{2} \cos \Delta \varphi, & \text { case (a) } \\
\frac{a_{1}+\varrho_{21} a_{2}}{2\left(1+\varrho_{21}\right)} \cos \Delta \varphi, & \text { case (b) }\end{cases}\right.
$$

where $\iota$ is the length of the true map feature for case (a). $\iota_{1}$ and $\iota_{2}$ are the lengths of two segments of the true map feature split by its point of intersection with the estimated map feature for case (b). $\varrho_{21}$ is the ratio between $\iota_{2}$ and $\iota_{1}$, which is denoted as $\iota_{2} / \iota_{1} . \Delta \varphi$ is the intersection angle between the estimated map feature and the true map feature. As shown in (23), the proposed error metric is independent of the origin position and the length of the reflector. In addition, it resolves the issue of different units for $\varphi$ and $\rho$. In other words, the proposed error metric only depends on the relative position and intersection angle between the estimated map feature and the true map feature. The proposed error metric takes into account both the displacement and the orientational error. The derivation of the error metric is shown in Appendix B.

\section{RESUlts}

This section compares the performance of the proposed MRMSC-PHD filter and several indoor tracking and mapping algorithms based on RFS theory, including the LMSC-PHD filter proposed in [25] and the multi-hypothesis SC-PHD filter (MH-SC-PHD filter) proposed in [27]. As shown in Fig. 1, the experimental environment was a half-enclosed area in the INFINITUS lab at the School of Electrical and Electronic Engineering, Nanyang Technological University (NTU), with dimensions of $14.5 \mathrm{~m} \times 5.7 \mathrm{~m}$. Three RDs were placed at known positions with coordinates $(3,3),(3,6.7)$, and $(8.9,6.7)$. The trajectory of the MD consisted of 49 points spaced by $20 \mathrm{~cm}$, starting from the upper right corner. The MD movement model and the TOA measurement model shown in Section II were used for all of the filters. The detection probability of the LOS and reflections was set as 0.9 [25]. The ceiling, floor, and pillar shown in Fig. 1 were used to generate the clutter measurement, and the clutter rate was set as 0.1 . The number of particles used to model the MD was 200 and the maximum number of GM components for each particle was set at $N_{\max }^{G M}=12$. For the MRMSCPHD filter and the LMSC-PHD filter, the greedy partition approach proposed in Section III-E1 was implemented, with the cut-off distance $c=1 \mathrm{~m}$ and maximum number of partitions $N_{\max }^{P}=5$. For the MRMSC-PHD filters, we considered two cases: first, the double-reflection-incorporating MSC-PHD (DRMSC-PHD) filter, which incorporates the LOS path and single and double reflection paths; and second, the triple-reflection-incorporating MSC-PHD (TRMSC-PHD) filter, which incorporates the LOS path and single, double, and triple reflection paths. To allow for fair comparison with the MH-SC-PHD filter, the number of hypotheses retained at each step was 200, and each hypothesis could generate 5 new hypotheses at the next step.

\section{A. Simulation Results}

The performance of the proposed filters under different measurement noises $\sigma_{z}^{l}$ and $\sigma_{z}^{m}$ was examined, where $\sigma_{z}^{l}$ and

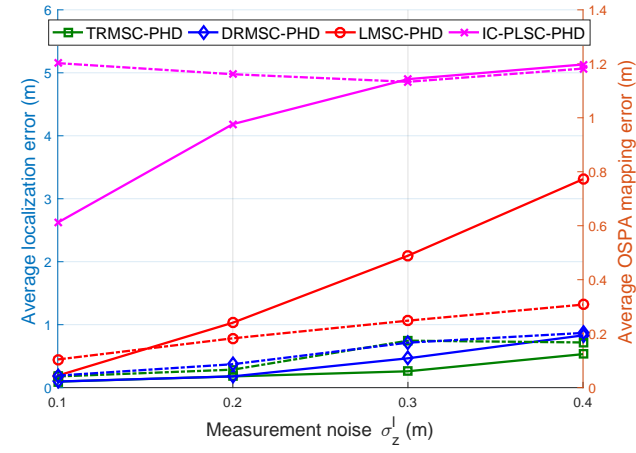

Fig. 6. Comparison of tracking and mapping performance with different measurement noise $\sigma_{z}^{l}$ : (a) Solid lines, average localization error (ALE) of MD, (b) Dashed lines, average OSPA mapping error (OSPA-AME) of map.

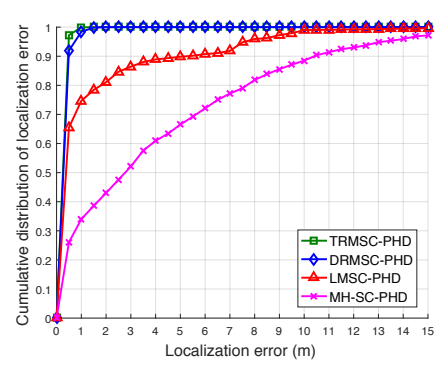

(a)

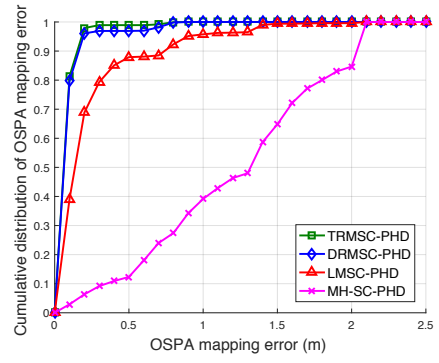

(b)
Fig. 7. Comparison of the tracking and mapping performance with uniformly distributed initial MD: (a) CDF of localization error, (b) CDF of OSPA mapping error.

$\sigma_{z}^{m}$ are as defined in (6) and represent the standard deviation of the LOS path and the multi-reflections, respectively, and $m$ is the order of reflection. In the simulations, $\sigma_{z}^{l}$ varied from $0.1 \mathrm{~m}$ to $0.4 \mathrm{~m}$. To account for reflection loss, we assumed $\sigma_{z}^{m}$ to be double for each reflection, which means that $\sigma_{z}^{m}=2^{m} \sigma_{z}^{l}$. Each filter was reiterated 25 times using different random sequences with perfect initialization of the MD and map features. The average localization error (ALE) of the MD and average OSPA mapping error (OSPA-AME) are presented in Fig. 6. As can be seen, the proposed TRMSC-PHD filter achieved ALE ranging from $0.1 \mathrm{~m}$ to $0.5 \mathrm{~m}$ and OSPA-AME ranging from $0.04 \mathrm{~m}$ to $0.17 \mathrm{~m}$ when $\sigma_{z}^{l}$ varied from $0.1 \mathrm{~m}$ to $0.4 \mathrm{~m}$. Meanwhile, the proposed DRMSC-PHD filter achieved ALE ranging from $0.1 \mathrm{~m}$ to $0.8 \mathrm{~m}$ and OSPA-AME ranging from $0.04 \mathrm{~m}$ to 0.20 $\mathrm{m}$ when $\sigma_{z}^{l}$ varied from $0.1 \mathrm{~m}$ to $0.4 \mathrm{~m}$. When $\sigma_{z}^{l}=0.4 \mathrm{~m}$, the proposed TRMSC-PHD filter outperformed the LMSCPHD and MH-MSC-PHD filters by around $85 \%$ and $90 \%$, respectively, in terms of localization accuracy. The proposed TRMSC-PHD filter also outperformed the LMSC-PHD and MH-MSC-PHD filters in terms of mapping accuracy by around $40 \%$ and $85 \%$, respectively.

The performance of the proposed filters under an inaccurate initial MD position was simulated. In the simulation, the measurement noise of the LOS path $\sigma_{z}^{l}=0.2 \mathrm{~m}$ and all other parameters remained the same as before. Each filter was reiterated 25 times using different random sequences, and the initial MD position was uniformly distributed in a $1 \mathrm{~m} \times 1 \mathrm{~m}$ area around the exact MD position. The cumulative 


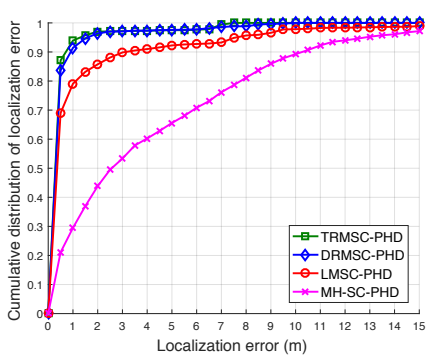

(a)

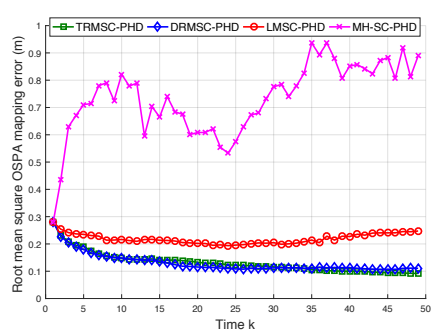

(c)

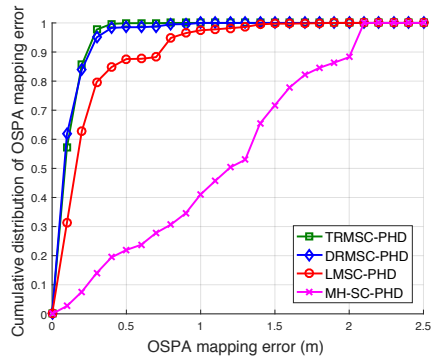

(b)

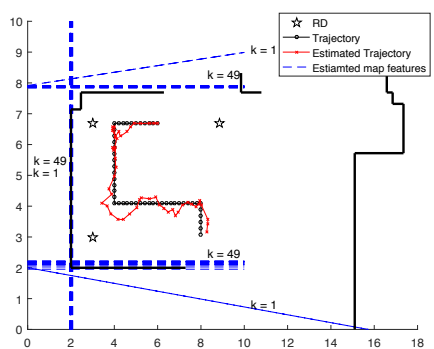

(d)
Fig. 8. Comparison of tracking and mapping performance with $\sigma_{\varphi}=5^{\circ}$ and $\sigma_{\rho}=0.1 \mathrm{~m}$, (a) CDF of localization error, (b) CDF of OSPA mapping error, (c) RMS mapping error along the trajectory, (d) Estimated trajectory and map features, where $k$ is the number of time steps.

distribution function (CDF) performance of the localization error (LE) with $0.5 \mathrm{~m}$ step-size and OSPA mapping error (OSPA-ME) with $0.1 \mathrm{~m}$ step-size are shown in Fig. 7. As can be seen, the proposed TRMSC-PHD and DRMSC-PHD filters achieved similar performances, with LE and OSPA-ME of around $0.4 \mathrm{~m}$ and $0.2 \mathrm{~m}$, respectively, $90 \%$ of the time. The results show that our proposed filter outperformed the LMSCPHD and MH-MSC-PHD filters in terms of localization accuracy by around $90 \%$ and $95 \%$, respectively. Our proposed filter also outperformed the LMSC-PHD and MH-MSC-PHD filters in terms of mapping accuracy by around $70 \%$ and $90 \%$, respectively. Note that the LMSC-PHD and MH-SCPHD filters have errors greater than $2 \mathrm{~m}$. This highlights the fact that, because these two filters can only handle LOS paths and single reflections, while considering all higher-order reflection paths as clutter, they perform poorly under heavily multipath situations.

Next, the performance of the proposed filters under an inaccurate initial map was simulated. In the simulation, the measurement noise of the LOS path $\sigma_{z}^{l}=0.2 \mathrm{~m}$ and all other parameters remained the same as before. Each filter was reiterated 25 times using different random sequences, and each time, the initial map was generated randomly according to the Gaussian distribution with $\sigma_{\varphi}=5^{\circ}$ and $\sigma_{\rho}=0.1 \mathrm{~m}$. The cumulative distribution function (CDF) performances of the localization error (LE) and OSPA mapping error (OSPA$\mathrm{ME)}$ are shown in Fig. 8. As can be seen, the proposed TRMSC-PHD and DRMSC-PHD filters achieved similar performances, with LE and OSPA-ME of around $0.7 \mathrm{~m}$ and 0.3 $\mathrm{m}$, respectively, $90 \%$ of the time. Therefore, our proposed filter outperformed the LMSC-PHD and MH-MSC-PHD filters in terms of localization accuracy by around $70 \%$ and $90 \%$,

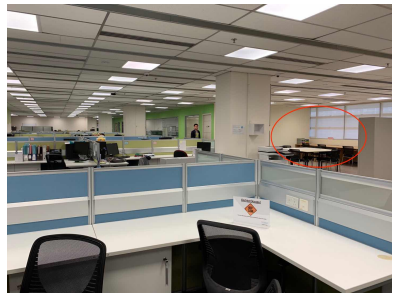

(a)

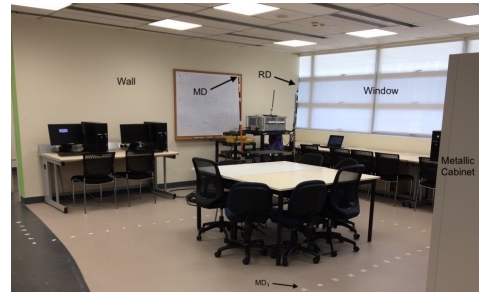

(b)
Fig. 9. Experimental environment, (a) View of the whole laboratory environment. (b) A snapshot of the experiment environment, which is a zoomed-in view of the area circled in red.

respectively. Our proposed filter also outperformed the LMSCPHD and MH-MSC-PHD filters in terms of mapping accuracy by around $55 \%$ and $85 \%$, respectively. We also studied how the root mean square OSPA-ME (RMS-OSPA-ME) changed along the trajectory, as shown in Fig. 8(c). As can be seen, the RMS-OSPA-ME of the proposed TRMSC-PHD and DRMSCPHD filters decreased continually from $0.3 \mathrm{~m}$ to $0.1 \mathrm{~m}$, showing stability and convergence. However, the RMS-OSPAME of the other filters either diverged or remained the same. For illustration, the estimated trajectory and map features of TRMSC-PHD filter based on 1 run data out of the total 25 runs data are shown in Fig. 8(d). As can be seen, at time $k=1$, the map features are initialized incorrectly. As time elapses, the map features become more accurate.

\section{B. Experimental Results}

A measurement campaign was carried out in a typical laboratory environment, as shown in Fig. 9. Fig. 9(a) shows the whole laboratory, with a height of $2.6 \mathrm{~m}$, in which the red circle highlights the experiment area for our proposed scheme. Fig. 9(b) shows a zoomed-in view of the experiment area. As can be seen, it contains a wall, a metallic cabinet, a window, a pillar, tables, ceiling, and floor. The MD and RDs are set at the same height of $1.9 \mathrm{~m}$. The $2 \mathrm{D}$ plane containing MD and RDs is shown in Fig. 1, and does not include the ceiling or floor. This experiment setting was chosen to test the performance and reliability of our proposed 2D scheme for indoor environments. The channel response between the MD and three RDs was measured using a single-input single-output system, the Agilent Technologies PNA-X Network Analyzer $\mathrm{N} 5244 \mathrm{~A}$, in a frequency domain with $500 \mathrm{MHz}$ bandwidth centered at $2.4 \mathrm{GHz}$ over 201 frequency points [13]. These channel responses, which included propagation paths reflected from the modeled walls as well as the pillar, ceiling, floor etc., were then used to estimate the TOA using an expectation maximization (EM) algorithm [43]. All propagation paths extracted from the EM were assumed to arise from the same 2D plane. Note that only paths associated with the modeled walls were used to track the MD and update the map features, and all of the remaining paths including those from the ceiling and floor were treated as clutter. For each snapshot, the number of multipaths estimated at each RD was 10, which included the LOS, single and higher-order reflection paths, and clutter. 


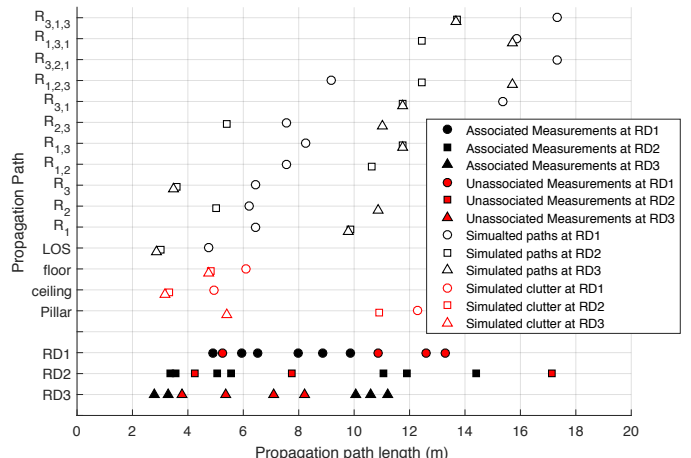

Fig. 10. Comparison of the measured and simulated reflection paths from walls and other reflectors such as the ceiling, floor, and pillar at $M D_{1}$.
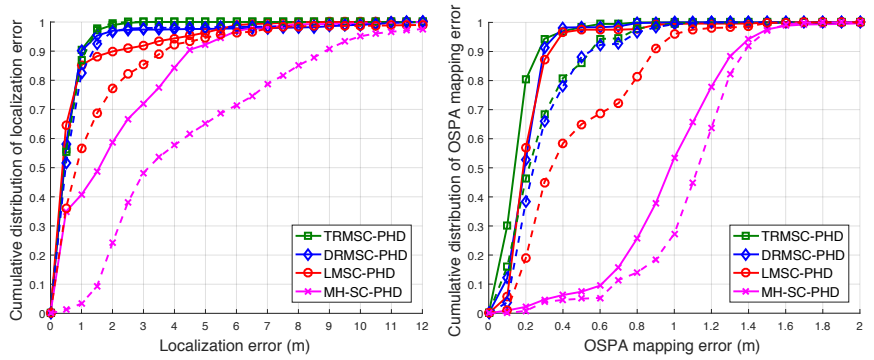

(a) Solid lines: three RDs; Dashed (b) Solid lines: three RDs; Dashed lines: $R D_{1}$ and $R D_{2}$

lines: $R D_{1}$ and $R D_{2}$

Fig. 11. Comparison of tracking and mapping performance based on collected measurement data using different numbers of RDs: (a) CDF performance of localization error, (b) CDF performance of OSPA mapping error.

To examine the quality of the measurements, Fig. $10 \mathrm{com}-$ pares the measurements at $M D_{1}$ with the simulated reflection paths from the walls, ceiling, floor, and pillar. Note that we only include three wall reflectors in our model. This means that the simulated reflections from the walls are considered as paths, and are denoted as $R_{i, j, l}$ in Fig. 10, where the subscript represents the collection of wall-reflected signals. The simulated reflections from the ceiling, floor, and pillar are considered as clutter. The measurements associated with the simulated paths are denoted as associated measurements, and the rest are referred to as unassociated measurements. The associated and unassociated measurements can be considered true measurements and clutter, respectively. As can be seen in Fig. 10, some of the unassociated measurements were in close proximity to the simulated clutter from the ceiling, floor, and pillar, and can be considered to have been generated by those features. This shows that the proposed MRMSC-PHD filter utilizes dominant multipath components and partial inaccurate map information to jointly localize the MD and correct the inaccurate map features.

Each filter was reiterated 25 times using different random sequences and initialization with perfect knowledge of the MD and map features. The CDF performances in terms of LE and OSPA-ME are shown as solid lines in Fig. 11. As can be seen, the proposed TRMSC-PHD and DRMSC-PHD filters achieved similar performance, with LE and OSPA-ME of around $1 \mathrm{~m}$ and $0.3 \mathrm{~m}$, respectively, $90 \%$ of the time. Our proposed filter outperformed the LMSC-PHD filter by $30 \%$ for LE. However,

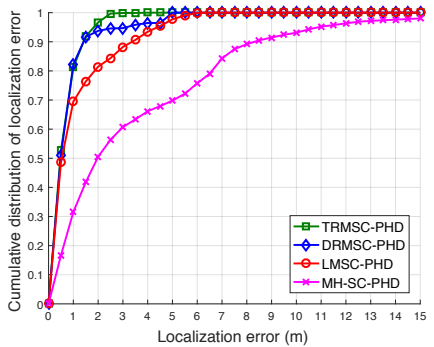

(a)

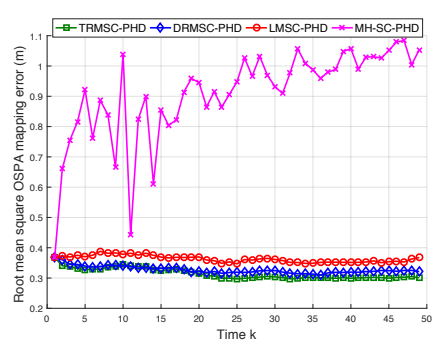

(c)

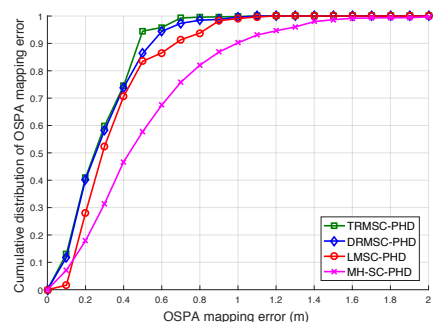

(b)

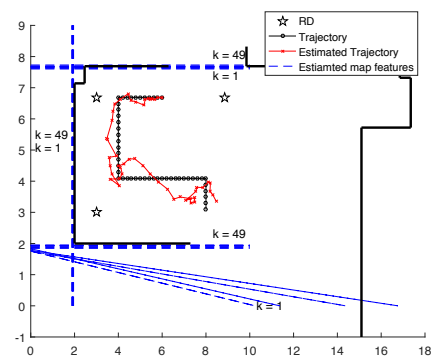

(d)
Fig. 12. Comparison of tracking and mapping performance with $\sigma_{\varphi}=5^{\circ}$ and $\sigma_{\rho}=0.1 \mathrm{~m}$ using all three RDs: (a) CDF of localization error, (b) CDF of OSPA mapping error, (c) RMS mapping error along the trajectory, (d) Estimated trajectory and map features.

the proposed filter showed little performance gain over the LMSC-PHD filter in terms of OSPA-ME, because in this semi-open cubical environment, single reflections are more numerous than higher-order reflections. Therefore, there are few higher-order reflections to further improve the OSPA-ME margin over the LMSC-PHD filter. However, in many indoor environments, there are often more higher-order reflections than single reflections [32]. This situation can be emulated by decreasing the number of RDs in the environment. The dashed lines in Fig. 11 show the performance of our proposed filter with fewer RDs. As can be seen, when using only $R D_{1}$ and $R D_{2}$, the proposed TRMSC-PHD and DRMSC-PHD filters achieved similar performance, with LE and OSPA-ME of around $1.2 \mathrm{~m}$ and $0.5 \mathrm{~m}$, respectively, $90 \%$ of the time. The results show that our proposed filter outperformed the LMSCPHD and MH-MSC-PHD filters in terms of localization accuracy by around $65 \%$ and $70 \%$, respectively. Our proposed filter also outperformed the LMSC-PHD and MH-MSC-PHD filters in terms of mapping accuracy by around $45 \%$ and $65 \%$, respectively. Thus, as the number of RDs decreases, which results in the number of detected single reflections decreasing, the performance margin between the proposed MRMSC-PHD filter and other filters increases. A performance comparison using only a single RD was also made, and showed an even larger performance margin; the details are omitted here for conciseness.

The performance of the proposed filters using measurement data under an inaccurate initial map was also examined. Each filter was reiterated 25 times using different random sequences, and each time, an initial map was generated randomly according to the Gaussian distribution with $\sigma_{\varphi}=5^{\circ}$ and $\sigma_{\rho}=0.1$ $\mathrm{m}$. The CDF performance for LE and OSPA-ME is shown 
in Fig. 12. As can be seen, the proposed TRMSC-PHD and DRMSC-PHD filters achieved similar performance, with LE and OSPA-ME of around $1.4 \mathrm{~m}$ and $0.5 \mathrm{~m}$, respectively, $90 \%$ of the time. The results show that our proposed filter outperformed the LMSC-PHD and MH-MSC-PHD filters in terms of localization accuracy by around $55 \%$ and $80 \%$, respectively. Our proposed filter also outperformed the LMSCPHD and MH-MSC-PHD filters in terms of mapping accuracy by around $30 \%$ and $50 \%$, respectively. We also examined how the RMS-OSPA-ME changed along the trajectory, as shown in Fig. 12(c). As can be seen, the RMS-OSPA-ME of the proposed TRMSC-PHD and DRMSC-PHD filters decreased continually from $0.37 \mathrm{~m}$ to $0.30 \mathrm{~m}$, showing stability and convergence. However, the RMS-OSPA-ME of the other filters either diverged or remained the same throughout. The estimated trajectory and map features are shown in Fig. 12(d), where $k$ is the number of time steps. As can be seen, at time $k=1$, the map features are initialized incorrectly. As time elapses, the map features become more accurate.

The computation time of the proposed MRMSC-PHD filters was also examined. Using a laptop with an Intel Xeon CPU E5-1650 v2 at $3.5 \mathrm{GHz}$ and MATLAB 2016a, the proposed MRMSC-PHD filter, which considers up to triple reflection paths, takes around 6.8 seconds to localize the MD and estimate the map features for each time step. The proposed MRMSC-PHD filter, which considers up to double reflection paths, takes around 4.2 seconds for each time step, and the LMSC-PHD filter takes around 3.7 seconds. If the algorithms were implemented on an FPGA, the computation time would be at least 10 times less [44]. The results presented in this section demonstrate that the proposed MRMSC-PHD filter can be successfully implemented in a real indoor environment to localize the MD and estimate the map features.

\section{CONCLUSiON}

This paper proposes a new MRMSC-PHD filter incorporating both the LOS and multiple reflections, which greatly enhances the accuracy of localization and tracking in indoor environments. A new greedy measurement partition scheme is designed to implement the MRMSC-PHD filter, and a novel error metric applicable to non-point scatterers is proposed to evaluate the error in the estimated map features. Based on simulation and experimental results, the proposed MRMSCPHD filter outperforms existing RFS-based algorithms in terms of LE and OSPA-ME by a significant margin. These results show the critical importance of including higher-order reflections in indoor tracking and mapping.

\section{APPENDIX A}

\section{UPDATE FORMULA OF MRMSC-PHD FILTER}

The joint update formula involves a set integral that is computationally intractable. Define the two-variable probabilitygenerating functionals (PGFLs) [26]

$$
\begin{aligned}
F[g, h]=\iint & h^{M_{k}} \prod_{s} G_{k}^{s}\left[g_{s} \mid \mathbf{x}_{\mathbf{k}}, M_{k}\right] p_{k \mid k-1}\left(\mathbf{x}_{\mathbf{k}} \mid Z_{1: k-1}\right) \\
& \times p_{k \mid k-1}\left(M_{k} \mid \mathbf{x}_{\mathbf{k}}, Z_{1: k-1}\right) \mathrm{d} \mathbf{x}_{\mathbf{k}} \delta M_{k}
\end{aligned}
$$

where $G_{k}^{s}\left[g_{s} \mid \mathbf{x}_{\mathbf{k}}, M_{k}\right]$ is the PGFL of $L_{k}\left(Z_{k, s} \mid \mathbf{x}_{\mathbf{k}}, M_{k}\right)$, which is defined as

$$
G_{k}^{s}\left[g_{s} \mid \mathbf{x}_{\mathbf{k}}, M_{k}\right]=\int g_{s}^{Z_{k, s}} L_{k}\left(Z_{k, s} \mid \mathbf{x}_{\mathbf{k}}, M_{k}\right) \delta Z_{k, s}
$$

The measurements from each RD consist of three classes: LOS, single and double reflections, and clutter measurements, with corresponding PGFLs as $G_{k}^{l}\left[g_{s} \mid \mathbf{x}_{\mathbf{k}}\right], G_{k}^{r}\left[g_{s} \mid \mathbf{x}_{\mathbf{k}}, M_{k}\right]$, and $G_{k}^{c}\left[g_{s}\right]$, respectively. According to the properties of the PGFLs, the $G_{k}^{s}\left[g_{s} \mid \mathbf{x}_{\mathbf{k}}, M_{k}\right]$ can be extended from [33] by adding an LOS component as

$$
G_{k}^{s}\left[g_{s} \mid \mathbf{x}_{\mathbf{k}}, M_{k}\right]=G_{k}^{l}\left[g_{s} \mid \cdot\right]\left(G_{k}^{r}\left[g_{s} \mid \cdot\right]\right)^{M_{k}} G_{k}^{c}\left[g_{s}\right]
$$

where

$$
\begin{aligned}
& G_{k}^{l}\left[g_{s} \mid \cdot\right]=1-P_{d}^{l, s}+P_{d}^{l, s} p_{g}^{l, s}, G_{k}^{r}\left[g_{s} \mid \cdot\right]=\prod_{m} \prod_{t} G_{k}^{m}\left[g_{s, t} \mid \cdot\right] \\
& G_{k}^{m}\left[g_{s} \mid \cdot\right]=1-P_{d}^{m, s, t}+P_{d}^{m, s, t} p_{g}^{m, s, t}, G_{k}^{c}\left[g_{s}\right]=\exp ^{\lambda_{c}^{s} c^{s}\left[g_{s}\right]-\lambda_{c}^{s}}
\end{aligned}
$$

$G_{k}^{l}\left[g_{s} \mid \cdot\right]$ and $G_{k}^{r}\left[g_{s} \mid \cdot\right]$ are abbreviations of $G_{k}^{l}\left[g_{s} \mid \mathbf{x}_{\mathbf{k}}\right]$ and $G_{k}^{r}\left[g_{s} \mid \mathbf{x}_{\mathbf{k}}, \mathbf{m}\right]$, respectively. $G_{k}^{m}\left[g_{s, t} \mid \cdot\right]$ is an abbreviation of $G_{k}^{m}\left[g_{s, t} \mid \mathbf{x}_{\mathbf{k}}, \mathbf{m}, \mathbf{p}_{k, s}^{m-1, t}\right]$.

$$
\begin{gathered}
p_{g}^{l, s}=\int g_{s}(z) l_{z}^{l, s} \mathrm{~d} z, c^{s}\left[g_{s}\right]=\int g_{s}(z) c_{s}(z) \mathrm{d} z \\
p_{g}^{m, s, t}=\int g_{s}(z) l_{z}^{m, s, t} \mathrm{~d} z
\end{gathered}
$$

The clutter is modeled as a Poisson process. Substituting (26) and (27) into (24), we obtain

$$
\begin{gathered}
F[g, h]=p_{\mathbf{x}}\left[G_{k}^{c}\left[g_{s}\right] \prod_{s} G_{k}^{l}\left[g_{s} \mid \cdot\right] G_{\mathbf{m}}\left[h \prod_{s} G_{k}^{r}\left[g_{s} \mid \cdot\right] \mid \cdot\right]\right] \\
p_{\mathbf{x}}[\Phi[h]]=\int \Phi[h] p_{k \mid k-1}\left(\mathbf{x}_{\mathbf{k}} \mid Z_{1: k-1}\right) \mathrm{d} \mathbf{x}_{\mathbf{k}}
\end{gathered}
$$

$G_{\mathbf{m}}[h \mid \cdot]$ is an abbreviation of $G_{\mathbf{m}}\left[h \mid \mathbf{x}_{\mathbf{k}}\right]$, the PGFL of the map features, which is defined based on the Poisson process assumption as

$$
\begin{gathered}
G_{\mathbf{m}}[h \mid \cdot]=\int h^{M_{k}} p_{k \mid k-1}\left(M_{k} \mid \mathbf{x}_{\mathbf{k}}, Z_{1: k-1}\right) \delta M_{k}=\exp ^{\mu p_{\mathbf{m}}\left[h \mid \mathbf{x}_{\mathbf{k}}\right]-\mu} \\
p_{\mathbf{m}}\left[h \mid \mathbf{x}_{\mathbf{k}}\right]=\int h(\mathbf{m}) p_{k \mid k-1}\left(\mathbf{m}_{\mathbf{k}} \mid \mathbf{x}_{\mathbf{k}}, Z_{1: k-1}\right) \mathrm{d} \mathbf{m}
\end{gathered}
$$

where $\mu$ is the average number of predicted map features, which is defined as $\mu=\int v_{k \mid k-1}\left(\mathbf{m} \mid \mathbf{x}_{\mathbf{k}}\right) \mathrm{d} \mathbf{m}$. Substituting (30) and (28) into (29), we obtain

$$
\begin{gathered}
F[g, h]=p_{\mathbf{x}}\left[\prod_{s} G_{k}^{l}\left[g_{s} \mid \cdot\right] \exp ^{\Phi[g, h]}\right] \\
\Phi[g, h]=\mu p_{\mathbf{m}}\left[h \prod_{s} G_{k}^{r}\left[g_{s} \mid \cdot\right] \mid \cdot\right]-\mu+\sum_{s}\left(c^{s}\left[g_{s}\right]-\lambda_{c}^{s}\right)
\end{gathered}
$$

Then, the functional derivative of $F[g, h]$ to set $Z_{k}$ is

$$
\begin{aligned}
\frac{\delta F}{\delta Z_{k}}[g, h]=p_{\mathbf{x}} & {\left[\sum_{\mathcal{P} \sqsubset Z_{k}} \exp ^{\Phi[g, h]}\right.} \\
& \left.\times \prod_{W \in \mathcal{P}} \Phi[\cdot]_{W}\left(\prod_{s} G_{k}^{l}\left[g_{s} \mid \cdot\right]+\sum_{W \in \mathcal{P}} \frac{L_{W}^{l}[g]}{\Phi[\cdot]_{W}}\right)\right]
\end{aligned}
$$




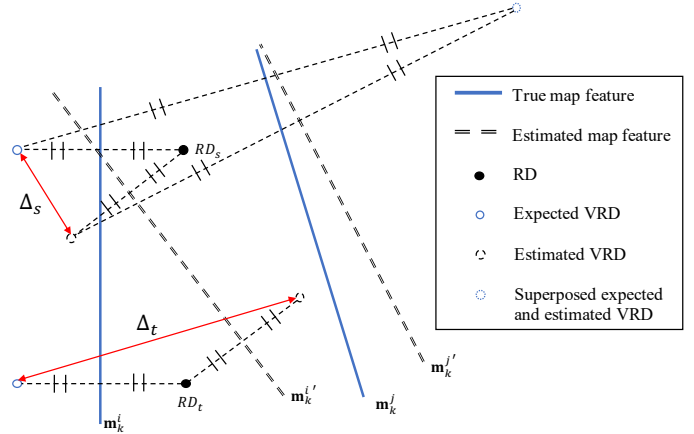

Fig. 13. Illustration of why the distance between the expected VRD and the estimated VRD cannot be used to determine the error in an estimated map feature.

where

$$
\begin{aligned}
L_{W}^{l}[g] & = \begin{cases}\prod_{s \in W} P_{d}^{l, s} l_{z_{s}}^{l, s} \prod_{s \notin W} G_{k}^{l}\left[g_{s} \mid \cdot\right], & \text { if } W_{s}=\left\{z_{s}\right\} \\
0, & \text { if }\left|W_{s}\right|>1 .\end{cases} \\
L_{W}^{r}[g] & =\prod_{s \nless W} G_{k}^{r}\left[g_{s} \mid \cdot\right] \\
& \times \prod_{s<W} \sum_{\theta_{s}}\left(\prod_{\theta_{s}(m, t)=0} G_{k}^{m}\left[g_{s, t} \mid \cdot\right] \prod_{\theta_{s}(m, t)>0} P_{d}^{m, s, t} l_{z_{\theta_{s}}, s, t}^{m, s}\right)
\end{aligned}
$$

Equation (32) can be proved by induction following the method of [45]. Based on the relationship between PGFL and PHD,

$$
v_{k \mid k}\left(\mathbf{x}_{\mathbf{k}}, \mathbf{m}\right)=\frac{\delta F}{\delta\left(\mathbf{x}_{\mathbf{k}}, \mathbf{m}\right) \delta Z_{k}}[0,1] / \frac{\delta F}{\delta Z_{k}}[0,1]
$$

Taking the functional derivative of $\frac{\delta F}{\delta Z_{k}}[0, h]$ to $\left(\mathbf{x}_{\mathbf{k}}, \mathbf{m}\right)$, we obtain

$$
\begin{aligned}
& \frac{\delta F}{\delta\left(\mathbf{x}_{\mathbf{k}}, \mathbf{m}\right) \delta Z_{k}}[0, h] \\
= & p_{k \mid k-1}\left(\mathbf{x}_{\mathbf{k}} \mid Z_{1: k-1}\right) \sum_{\mathcal{P} \subset Z_{k}} \exp ^{\Phi[0, h]}\left(1-\tilde{P}_{d}^{l}+\sum_{W \in \mathcal{P}} \frac{L_{W}^{l}}{\Gamma_{W}[h]}\right) \\
\times & \prod_{W \in \mathcal{P}} \Gamma_{W}[h]\left(1-\tilde{P}_{d}^{r}+\sum_{W \in \mathcal{P}}\left(1-\Omega_{W}[h]\right) \frac{L_{W}^{r}}{\Gamma_{W}[h]}\right) v_{k \mid k-1}\left(\mathbf{m} \mid \mathbf{x}_{\mathbf{k}}\right)
\end{aligned}
$$

where

$$
\begin{gathered}
\Omega_{W}[h]=\frac{L_{W}^{l} / \Gamma_{W}[h]}{1-\tilde{P}_{d}^{l}+\sum_{W \in \mathcal{P}}\left(L_{W}^{l} / \Gamma_{W}[h]\right)} \\
\Phi[0, h]=v_{k \mid k-1}\left[h\left(1-\tilde{P}_{d}^{r}\right) \mid \mathbf{x}_{\mathbf{k}}\right]-\mu-\sum_{s} \lambda_{c}^{s} \\
\Gamma_{W}[h]=\left.\Phi[\cdot]_{W}\right|_{g=0}=c_{W}+v_{k \mid k-1}\left[h L_{W}^{r} \mid \mathbf{x}_{\mathbf{k}}\right]
\end{gathered}
$$

Dividing (35) by $\frac{\delta F}{\delta Z_{k}}[0, h]$ and setting $h=1$, we obtain the update formulas of the MRMSC-PHD filter shown in (12).

\section{APPENDIX B}

\section{PROPOSED ERROR METRIC}

In this section, we will first show that the distance between the estimated VRD and the expected VRD is not appropriate to be used to represent the error in the map features because it depends on the relative position of the RD with respect to those features. It can be shown that different distances between the estimated and the expected VRD can produce identical errors in the map features. Fig. 13 shows two RDs denoted as $R D_{s}$ and $R D_{t}$, the true map feature $\mathbf{m}_{k}^{i}$, and the corresponding estimated map feature $\mathbf{m}_{k}^{i}{ }^{\prime}$. As can be seen, the distance between the estimated and the expected VRDs of $R D_{s}, \delta_{s}$, is smaller than the distance between the estimated and the expected VRDs of $R D_{t}, \delta_{t}$. This means that for a given distance between the estimated and the expected VRDs, we cannot infer the actual error between the estimated map feature and the true map feature. For higher-order VRDs, the distance between the expected and the estimated VRDs may be zero even if the map features are estimated inaccurately, as shown in Fig. 13.

Next we show the derivation of the proposed error metric. Note that the intersection angle between the estimated map feature and the true map feature cannot be greater than $\pi / 4$; otherwise, the estimated map feature will be assigned to another map feature with an intersection angle smaller than $\pi / 4$ by the OSPA metric. For case (a) in Figure 5 , the estimated map feature does not intersect with the true map feature but does intersect with the extension of the true map feature. Then, the enclosed trapezoidal area can be expressed as

$$
\begin{aligned}
A & =\frac{1}{2}\left(a_{1}+a_{2}\right) \iota \cos \Delta \varphi \\
& =\frac{1}{2}\left(\iota^{\prime} \sin \Delta \varphi+\left(\iota+\iota^{\prime}\right) \sin \Delta \varphi\right) \iota \cos \Delta \varphi \\
& =\frac{1}{4}\left(\iota^{2}+2 \iota^{\prime} \iota\right) \sin 2 \Delta \varphi
\end{aligned}
$$

As shown, for a fixed $\Delta \varphi, A$ increases as $i$ increases, which means that as the estimated map feature becomes further from the true map feature, the error increases. If $i$ becomes positive infinity, then $\Delta \varphi=0$, and the error only depends on $a_{1}$. For a fixed $\iota^{\prime}, A$ increases as $\Delta \varphi$ increases from 0 to $\pi / 4$, which means that as the estimated map feature becomes more tilted, the error likewise increases. Similarly, for case (b) in Figure 5, the estimated map feature intersects the true map feature at the boundary between segments $\iota_{1}$ and $\iota_{2}$. Then, the two enclosed triangular areas can be expressed as

$$
\begin{aligned}
A & =\left(A_{1}+A_{2}\right)=\frac{1}{2}\left(\iota_{1} a_{1}+\iota_{2} a_{2}\right) \cos \Delta \varphi \\
& =\frac{1}{4}\left(\iota_{1}^{2}+\iota_{2}^{2}\right) \sin 2 \Delta \varphi \\
& \leq \frac{1}{4}\left(\iota_{1}+\iota_{2}\right)^{2} \sin 2 \Delta \varphi=\frac{1}{4} \iota^{2} \sin 2 \Delta \varphi
\end{aligned}
$$

The equality holds when $\iota_{1}$ or $\iota_{2}$ is zero. As shown, for a fixed $\Delta \varphi, A$ increases as the intersection point moves from the center to the endpoint of the true map feature. For a fixed $\iota_{1}$ and $\iota_{2}$, which means a fixed intersection point, $A$ increases as $\Delta \varphi$ increases from 0 to $\pi / 4$, which means that as the estimated map feature becomes more tilted, the error increases.

As can be seen, for both cases, the area $A$ reflects the difference between the estimated map feature and the true map feature. Normalizing $A$ by the length of the true map feature, the proposed error metric can be derived. 


\section{REFERENCES}

[1] W. Xue, W. Qiu, X. Hua, and K. Yu, "Improved Wi-Fi RSSI measurement for indoor localization," IEEE Sensors J., vol. 17, pp. 2224 - 2230, Apr. 2017.

[2] F. Wen and C. Liang, "Fine-grained indoor localization using single access point with multiple antennas," IEEE Sensors J., vol. 15, pp. 1538 - 1544, Mar. 2015

[3] S. W. Chen, S. Y. Tan, and C. K. Seow, "Peer-to-peer localization in urban and indoor environments," Progress In Electromagnetics Research $B$, vol. 33, pp. $339-358,2011$.

[4] X. Tian, W. Li, Y. Yang, Z. Zhang, and X. Wang, "Optimization of fingerprints reporting strategy for WLAN indoor localization," IEEE Trans. Mobile Comput., vol. 17, pp. 390 - 403, Feb. 2018.

[5] S. Lanzisera, D. Zats, and K. S. J. Pister, "Radio frequency time-offlight distance measurement for low-cost wireless sensor localization," IEEE Sensors J., vol. 11, pp. 837 - 845, Mar. 2011.

[6] Z. Li, Z. Tian, M. Zhou, Z. Zhang, and Y. Jin, "Awareness of line-ofsight propagation for indoor localization using hopkins statistic," IEEE Sensors J., vol. 18, pp. 3864 - 3874, May 2018.

[7] K. Yu and E. Dutkiewicz, "NLOS identification and mitigation for mobile tracking," IEEE Trans. Aerosp. Electron. Syst., vol. 49, pp. 1438 - 1452, Jul. 2013.

[8] S. W. Chen, C. K. Seow, and S. Y. Tan, "Elliptical Lagrange-based NLOS tracking localization scheme," IEEE Trans. Wireless Commun., vol. 15, pp. 3212 - 3225, May 2016.

[9] H. Zhang, C. K. Seow, and S. Y. Tan, "Virtual reference device-based narrowband TOA localization using LOS and NLOS path," in Proc. IEEE/ION Position Location and Navigation Symposium (PLANS'16), Savannah, GA, Apr. 11-14, 2016, pp. 225 - 231.

[10] C. K. Seow and S. Y. Tan, "Non-line-of-sight unidirectional mobile localisation in multipath environment," Electron. Lett., vol. 44, pp. 141 - 142, Jan. 2008.

[11] E. Leitinger, M. Fröhle, P. Meissner, and K. Witrisal, "Multipath-assisted maximum-likelihood indoor positioning using UWB signals," in Proc. IEEE Int. Conf. Communications Workshops (ICCW'14), Sydney, NSW, Jun. 10-14, 2014, pp. $170-175$

[12] S. W. Chen, C. K. Seow, and S. Y. Tan, "Virtual reference devicebased NLOS localization in multipath environment," IEEE Antennas and Wireless Propag. Lett., vol. 13, pp. 1409-1412, Jul. 2014.

[13] S. Y. Tan and H. S. Tan, "Improved three-dimensional ray tracing technique for microcellular propagation models," Electron Lett., vol. 31, pp. 1503 - 1505, Aug. 1995.

[14] Y. Bar-Shalom and T. E. Fortmann, Tracking and Data Association. San Diego, CA: Academic Press, 1988.

[15] D. Musicki and R. Evans, "Joint integrated probabilistic data association: JIPDA,” IEEE Trans. Aerosp. Electron. Syst., vol. 40, pp. 1093 - 1099, Jul. 2004.

[16] Z. Chen, H. Zou, H. Jiang, Q. Zhu, Y. C. Soh, and L. Xie, "Fusion of wifi, smartphone sensors and landmarks using the kalman filter for indoor localization," Sensors, vol. 15, pp. 715 - 732, Jan. 2015.

[17] D. Gustafson, J. Elwell, and J. Soltz, "Innovative indoor geolocation using rf multipath diversity," in Proc. IEEE/ION Position Location and Navigation Symposium (PLANS'06), Coronado, CA, Apr. 25-27, 2006, pp. $904-912$

[18] S. Blackman, Multiple Target Tracking with Radar Applications. Norwood, MA: Artech House, 1986.

[19] R. L. Streit and T. E. Luginbuhl, "Maximum likelihood method for probabilistic multi-hypothesis tracking," in Proc. SPIE, Signal and Data Process. of Small Targets, Orlando, FL, jul. 6, 1994, pp. 5 - 7.

[20] L. Li and J. L. Krolik, "Simultaneous target and multipath positioning," IEEE J. Sel. Topics Signal Process, vol. 8, pp. 153-165, Feb. 2014

[21] S. Blackman, "Multiple hypothesis tracking for multiple target tracking," IEEE Aerosp. Electron. Syst. Mag., vol. 19, pp. 5-18, Jan. 2004.

[22] J. Niera and J. D. Tardos, "Data association in stochastic mapping using the joint compatibility test," IEEE Trans. Robot. Autom., vol. 17, pp. 890 - 897, Dec. 2001.

[23] J. Mullane, B.-N. Vo, M. D. Adams, and B.-T. Vo, "A random-finite-set approach to Bayesian SLAM," IEEE Trans. Robot., vol. 27, pp. 268 282, Apr. 2011.

[24] J. Mullane, B.-N. Vo, M. D. Adams, and W. S. Wijesoma, "A random set formulation for Bayesian SLAM," in Proc. IEEE/RSJ Int. Conf. Intell. Robot. Syst. (IROS'08), Nice, France, Sept. 22-26, 2008, pp. 1043 1049.

[25] H. Zhang and S. Y. Tan, "TOA based indoor localization and tracking via single-cluster PHD filtering," in Proc. IEEE Global Commun. Conf. (Globecom'17), Singapore, Dec. 4-8, 2017, pp. 1 - 6.
[26] C. S. Lee, D. E. Clark, and J. Salvi, "SLAM with dynamic targets via single-cluster PHD filtering," IEEE J. Sel. Topics Signal Process, vol. 7, pp. $543-552$, Jun. 2013.

[27] L. Li and J. L. Krolik, "Target tracking in uncertain multipath environment using distributed angle-of-arrival observation," in Proc. IEEE Radar Conference (RadarCon'15), Arlington, VA, May. 10-15, 2015, pp. $1473-1478$

[28] C. S. Lee, D. E. Clark, and J. Salvi, "SLAM with single cluster PHD filters," in Proc. IEEE Int. Conf. Robot. Autom. (ICRA'12), Saint Paul, MN, may. 14-18, 2012, pp. 2096 - 2101.

[29] R. P. S. Mahler, "Multitarget Bayes filtering via first-order multitarget moments," IEEE Trans. Aerosp. Electron. Syst., vol. 39, pp. 1152 - 1178, Oct. 2003.

[30] B.-N. Vo and W.-K. Ma, "The Gaussian mixture probability hypothesis density filter," IEEE Trans. Signal Process., vol. 54, pp. 4091 - 4104, Nov. 2006.

[31] R. P. S. Mahler, Statistical Multisourse-Multitarget Information Fusion. Norwood, MA: Artech House, 2007.

[32] S. Y. Tan and H. S. Tan, "Modelling and measurements of channel impulse response for an indoor wireless communication system," IEE Proc.-Microw. Antennas Propag., vol. 142, pp. 405 - 410, Oct. 1995.

[33] X. Tang, X. Chen, M. McDonald, R. Mahler, R. Tharmarasa, and T. Kirubarajan, "A multiple-detection probability hypothesis density filter," IEEE Trans. Signal Process., vol. 63, pp. 2007 - 2019, Apr. 2015.

[34] X. Shen, Z. Song, H. Fan, and Q. Fu, "Generalised distance partitioning for multiple-detection tracking filter based on random finite set," IET Radar, Sonar \& Navigation, vol. 12, pp. 260 - 267, Feb. 2018.

[35] R. P. S. Mahler, "The multisensor PHD filter, II: Erroneous solution via Poisson magic," in Proc. SPIE, Signal Process., Sensor Fusion, and Target Recognit. XVIII, Orlando, FL, may. 11, 2009, pp. 73 360D1 $73360 \mathrm{D} 12$.

[36] S. Nagappa and D. E. Clark, "On the ordering of the sensors in the iterated-corrector probability hypothesis density (PHD) filter," in Proc. SPIE, Signal Process., Sensor Fusion, and Target Recognit. XX, Orlando, FL, may. 5, 2011, pp. 80500M-1 - 80500M-6.

[37] K. Granström, C. Lundquist, and O. Orguner, "Extended target tracking using a Gaussian-mixture PHD filter," IEEE Trans. Aerosp. Electron. Syst., vol. 48, pp. 3268-3286, Oct. 2012

[38] S. Nannuru, S. Blouin, M. Coates, and M. Rabbat, "Multisensor CPHD filter," IEEE Trans. Aerosp. Electron. Syst., vol. 52, pp. 1834 - 1854, Aug. 2016.

[39] A.-A. Saucan, M. J. Coates, and M. Rabbat, "A multisensor multiBernoulli filter," IEEE Trans. Signal Process., vol. 65, pp. 5495 - 5509, Oct. 2017.

[40] D. Clark and R. P. S. Mahler, "Generalized PHD filters via a general chain rule," in Proc. 15th Int. Conf. Inf. Fusion (Fusion'12), Singapore, jul. 9-12, 2012, pp. 157-164.

[41] D. Schuhmacher, B.-T. Vo, and B.-N. Vo, "A consistent metric for performance evaluation of multi-object filters," IEEE Trans. Signal Process., vol. 56, pp. 3447 - 3457, Aug. 2008.

[42] H. Naseri and V. Koivunen, "Cooperative simultaneous localization and mapping by exploiting multipath propagation," IEEE Trans. Signal Process., vol. 65, pp. 200 - 211, Jan. 2017.

[43] K. Haneda and J.-I. Takada, "An application of SAGE algorithm for UWB propagation channel estimation," in IEEE Conf. Ultra Wideband Systems and Technologies (UWST'03), Reston, VA, nov. 16 - 19, 2003, pp. $483-487$.

[44] C. Cullinan, C. Wyant, T. Frattesi, and X. Huang, "Computing performance benchmarks among CPU, GPU, and FPGA," Internet: www. wpi. edu/Pubs/E-project/Available/E-project-030212123508/unrestricted/Benchmarking Final, 2013.

[45] R. P. S. Mahler, "The multisensor PHD filter, I: General solution via multitarget calculus," in Proc. SPIE, Signal Process., Sensor Fusion, and Target Recognit. XVIII, Orlando, FL, may. 11, 2009, pp. 73 360E1 $-73360 \mathrm{E} 12$. 